\title{
Quand les technologies nomades influencent simultanément le bien-être et le stress au travail
}

\section{When nomadic technologies simultaneously influence well- being and stress at work}

\author{
Pierre Loup \\ MRM, université de Montpellier, Montpellier, France \\ pierre.loup@umontpellier.fr \\ Jonathan Maurice \\ TSM-Research, université Toulouse Capitole, CNRS, France \\ jonathan.maurice@tsm-education.fr \\ Florence Rodhain \\ MRM, université de Montpellier, Montpellier, France \\ florence.rodhain@umontpellier.fr
}

Pour citer cet article :

LOUP (Pierre), MAURICE (Jonathan) et RODHAIN (Florence), « Quand les technologies nomades influencent simultanément le bien-être et le stress au travail », Systèmes d'information et management, décembre 2020, vol. 25, n 3, p. 9-49 ; https://doi.org/10.3917/sim.203.0009. 


\title{
Résumé :
}

L'objectif de cet article est d'étudier l'influence des technologies nomades (TN) sur le bienêtre et le stress des individus au travail. Deux questions de recherche sont envisagées : (1) Dans quelle mesure les individus perçoivent-ils ces technologies comme des exigences et/ou des ressources supplémentaires dans l'exercice de leur travail ? (2) Comment la combinaison des exigences et ressources liées aux TN influence-t-elle le bien-être, le stress et la performance au travail ? Pour répondre à ces questions, une étude qualitative a été menée au sein du groupe La Poste ayant introduit des TN pour ses directions commerciales. Les résultats montrent que ces dernières sont d'abord perçues comme des ressources par les individus, et entrainent une amélioration de leur bien-être au travail. Dans le même temps et indépendamment, elles engendrent une surcharge de travail et une invasion de la technologie dans la sphère personnelle, influençant le niveau de stress auquel peut être confronté l'individu. Sur le plan théorique, l'article met alors en exergue la déconnexion entre bien-être au travail et stress technologique, les deux pouvant émerger indépendamment lors de l'introduction de $\mathrm{TN}$, à travers des comportements d'addiction. Nous développons grâce aux résultats un modèle job demands-resources (JD-R) adapté à ce contexte et construisons un ensemble de propositions de recherche émergeant de notre cas.

Mots clés : technologies nomades, bien-être au travail, stress professionnel, bien-être technologique, technostress, modèle job demands-resources.

\begin{abstract}
The aim of this article is to analyze the role of mobile technologies (MT) on employees' wellbeing and technostress. Two research questions are considered: (1) To what extent do employees perceive these technologies as additional job demands and/or resources in their daily work? (2) How does the combination of demands and resources given by MT influence wellbeing, stress and performance at work? To answer these questions, a qualitative study was conducted within La Poste group that introduced MT for its commercial departments. The results show that this kind of devices is perceived as resources by individuals and improve their well-being at work. At the same time, and independently, they generate techno-overload and techno-invasion in private life, increasing the level of stress to which the individual may be exposed. From a theoretical perspective, this study documents the disconnection between wellbeing at work and technostress. Both concepts can be fostered independently and simultaneously by the introduction of MT, namely through addictive behaviors. Based on our results, we develop a job demands-resources model (JD-R) adapted to such an introduction and formulate a set of research propositions.
\end{abstract}

Keywords: mobile technologies, well-being at work, stress at work, techno-well-being technostress, job demands-resources model. 


\section{Introduction}

Les évolutions technologiques et l'omniprésence des technologies numériques au sein des organisations peuvent conduire à des changements organisationnels (Godé-Sanchez, 2008 ; Habib, Béjean et Dumond, 2017) et à une modification des contours des métiers, notamment par le développement de compétences spécifiques à l'utilisation des technologies. Ainsi, la diffusion technologique au sein d'une organisation peut s'accompagner d'un accroissement de la charge de travail et d'une intensification du travail avec un bouleversement du rythme (Bretesché, Corbière et Geffroy, 2012 ; Greenan et al., 2012 ; GRePS-Apec, 2011 ; Gupta, Li et Sharda, 2013 ; Isaac, Campoy et Kalika, 2007 ; Venin, 2013). En effet, la complémentarité plus que la substitution est de règle, les tâches amenées par les technologies de l'information et de la communication (TIC) ne viennent pas en remplacement mais constituent souvent un effet additif, engendrant ainsi un effet mille-feuilles (Boukef et Charki, 2019 ; Kalika, Charki et Isaac, 2007). Parmi ces évolutions technologiques, les technologies nomades (téléphones intelligents ${ }^{1}$, tablettes, ordinateurs ultralégers), qui permettent l'utilisation de données numériques de manière asynchrone, ont bouleversé l'organisation spatio-temporelle du travail (Besseyre des Horts et Isaac, 2006). Ainsi, elles influencent le rythme de travail nécessitant souvent une forme d'immédiateté dans les échanges et une simultanéité des actions ; elles influent sur l'organisation de la journée de travail en pesant notamment sur l'emploi du temps et en étant source de « repriorisation » des tâches à accomplir au fil de la journée ; elles génèrent une problématique de fractionnement des tâches et de morcèlement des temps et des espaces de travail pouvant conduire à une perte de sens du travail. Par exemple, Klein et Govaere (2012) rapportent que les managers américains sont sollicités toutes les 3 ou 4 minutes par les TIC (courriel, SMS...) pour effectuer des tâches diverses. Une étude menée par la société Adobe (2015) révèle quant à elle que les cadres français passent en moyenne plus de $5 \mathrm{~h}$ par jour sur leur messagerie électronique tandis que deux tiers des cadres européens regardent leurs emails professionnels en dehors des heures de travail. Des observations similaires sont relatées par l'enquête Webtorials (2017) révélant que la consultation de la messagerie électronique professionnelle représente le principal outil de communication en termes de temps passé. Très récemment, une tribune du journal Le Monde (Garo, 2019) affirme même que les technologies nomades renforcent les interruptions subies sur le lieu de travail. Malgré cela, les technologies nomades sont souvent perçues comme une récompense par les salariés car elles viennent notamment valoriser leur statut social. Il est aussi souvent considéré qu'elles améliorent nécessairement les conditions de travail (Centre d'analyse stratégique, 2012).

Ainsi, devant les différents enjeux sociaux et économiques accompagnant la diffusion de ces technologies, une étude des effets potentiels de ces dernières sur le niveau de bien-être au travail (BET) et de stress des salariés parait pertinente. Dans ce contexte, un travail d'investigation a été initié avec La Poste dont les équipes commerciales ont été équipées en technologies nomades (ordinateurs convertibles en tablettes et mobiles multifonctions) à la fin de l'année 2012. L'objectif de ce travail de recherche est ainsi d'étudier la manière dont est perçue l'introduction de ces technologies par l'ensemble des acteurs considérés et de comprendre comment les technologies nomades s'intègrent dans leur quotidien; il est alors possible de déterminer dans quelle mesure elles sont envisagées comme exigences ou ressources par les individus, en évaluant leur influence conjointe sur le bien-être et le stress au travail. Le modèle job demands-resources initié par Demerouti et al. (2001) puis révisé par Schaufeli et Bakker (2004) est adapté et utilisé pour comprendre l'influence des technologies nomades sur l'organisation et les individus.

\footnotetext{
${ }^{1}$ Les termes « mobiles multifonctions » et « téléphones intelligents » sont utilisés de façon interchangeable dans cet article.
} 
Dans la mesure où les technologies nomades (mobiles multifonctions, tablettes, ordinateurs portables, outils logiciels en ligne; notées TN dans l'article) ont tendance à bouleverser les rapports aux autres, aux temps et à l'espace de travail, nous étudions leur influence sur le BET, le stress et la performance, étant donné les transformations importantes engendrées par la mise en place de ces technologies dans les organisations. Autrement dit, nous cherchons à mettre en lumière comment les TN peuvent engendrer une situation de BET et/ou de stress pour les employés, et comment cela se traduit en termes de performances individuelle et organisationnelle. Plus précisément, cette étude qualitative vise à répondre aux deux questions de recherche suivantes.

$\mathrm{QR}_{1}$. Dans quelle mesure les individus perçoivent-ils les technologies nomades comme des exigences et/ou des ressources supplémentaires dans l'exercice de leur travail ?

$\mathrm{QR}_{2}$. Comment la combinaison des exigences et ressources liées aux $\mathrm{TN}$ influence-t-elle le bien-être, le stress et la performance au travail ?

Les réponses à ces questions de recherche contribuent à la littérature sur le bien-être et le stress au travail. Elles participent également à la littérature en systèmes d'information dans la mesure où l'on considère à l'instar de Reix et Rowe (2002, p. 11) que ces technologies font partie du système d'information des entreprises, à savoir "un ensemble d'acteurs sociaux qui mémorisent et transforment des représentations via des technologies de l'information et des modes opératoires $\gg$. Tout d'abord, l'étude qualitative permet d'identifier les couples exigences-ressources associés à l'introduction des TN en montrant qu'ils sont à la fois sources de stress et de bien-être au travail, engendrant une meilleure efficacité. Ensuite, la présence simultanée de stress et de bien-être au travail est évoquée par les individus avec, à l'extrême, des comportements d'addiction. Cela implique un découplage entre stress et bien-être technologique lors de l'introduction de TN qui nous conduit formuler des propositions de recherche et à développer d'un modèle JD-R adapté à l'introduction des TN.

La suite de l'article est organisée comme suit. Nous définissons en section 2 les notions de stress professionnel, de bien-être au travail, de stress technologique ou technostress ${ }^{2}$ et de bienêtre technologique pour détailler les influences positives et négatives des TN sur les individus. Nous introduisons ensuite le modèle job demands-resources (JD-R) révisé que nous adaptons à l'introduction des TN. En section 3, nous présentons la démarche de recherche mise en œuvre au sein du groupe La Poste. Nous exposons en section 4 les résultats de cette étude qualitative à travers le cadre théorique du modèle JD-R avant de discuter ces résultats pour aboutir à des propositions qualitatives en section 5 et de conclure l'étude en section 6 .

\section{L'influence conjointe des technologies nomades sur le bien-être et le stress au travail : une analyse par le modèle JD-R}

Caractéristiques de notre époque, les mutations technologiques et la diffusion intense des TN engendre des interactions individu-technologie qui selon le contexte organisationnel sont de nature à produire des effets négatifs (stress technologique) et des effets positifs (bien-être technologique). Ainsi, dans un premier temps, nous présentons les concepts généraux de stress professionnel et de bien-être au travail pour proposer ensuite une synthèse de la littérature mettant en évidence les effets négatifs (stress technologique) et positifs (bien-être technologique) des TN sur les plans individuel et organisationnel. Nous faisons enfin le lien

\footnotetext{
2 Nous nous focalisons ici uniquement sur les facteurs de stress liés aux technologies. Ces derniers sont habituellement appréhendés dans la littérature en systèmes d'information au travers du concept de technostress.
} 
entre cette littérature et le modèle JD-R révisé, ce dernier permettant d'envisager l'ambivalence entre ces deux notions qui peuvent coexister et se développer de façon simultanée.

\subsection{Concepts de stress professionnel et de bien-être au travail}

Le stress professionnel, qui correspond au stress provoqué ou induit par le contexte de travail, occupe une place centrale parmi les risques psychosociaux. La confusion conceptuelle et les divergences de points de vue qui entourent cette notion ont depuis longtemps conduit plusieurs auteurs à noter le manque de consensus autour de sa définition (Beehr et Newman, 1978 ; McGrath, 1976; Schuler, 1980). Ainsi, l'appréhension de la notion de stress revêt une connotation particulière selon la discipline dans laquelle elle s'inscrit. D'un point de vue médical, le stress est envisagé en tant que réponse, il constitue un stimulus pour les sociologues, alors que les psychologues le perçoivent au travers de l'interaction entre l'individu et son environnement (Koolhaas et al., 2011 ; Lazarus et Folkman, 1984 ; Légeron, 2015 ; Selye, 1976). Selon l'Agence européenne pour la santé et la sécurité au travail (2002), "un état de stress survient lorsqu'il y a déséquilibre entre la perception qu'une personne a des contraintes que lui impose son environnement et la perception qu'elle a de ses propres ressources pour y faire face ». Bien que le processus d'évaluation des contraintes et des ressources soit d'ordre psychologique, les effets du stress ne sont pas eux uniquement de même nature et peuvent également affecter la santé physique, le bien-être et la productivité.

Les divergences de points de vue quant à la définition du stress ont donné lieu au développement de nombreux modèles dans la littérature consacrée au sujet. Parmi les approches envisagées, deux d'entre elles se distinguent particulièrement : l'approche interactionniste (French et al., 1982 ; Karasek, 1979 ; Karasek, 1990) et l'approche transactionnelle (Lazarus et Folkman, 1984 ; Siegrist, 1996). L'approche interactionniste, qui prend également en compte les spécificités du travailleur et de son environnement, s'appuie sur le fait que, face à un évènement, les réactions sont différentes en fonction des individus. Le stress est alors le résultat de l'incompatibilité entre les caractéristiques et les attentes de l'individu et les éléments de l'environnement. Dans l'approche transactionnelle, le stress est lié à la transaction entre le travailleur et son environnement. Ainsi, la perception de la situation par l'individu joue un rôle prépondérant quant à l'apparition ou non d'un stress (Lazarus et Folkman, 1984). Quelle que soit l'approche, les recherches se focalisant uniquement sur le stress professionnel sont centrées sur la réparation et non la prévention de la santé psychologique. Elles adoptent ainsi une vision pathogénique qui est aujourd'hui de plus en plus complétée par une approche plus positive.

Cette dernière approche dite salutogénique cherche à se détacher de l'approche pathogénique focalisée sur les aspects négatifs de la santé au travail (Abord de Chatillon et Richard, 2015) en mettant au centre de son analyse le bien-être au travail. Soutenue conjointement par les praticiens et les chercheurs, cette approche salutogénique vise à favoriser le bien-être et la qualité de vie des employés sous un angle positif et préventif. Le BET s'inscrit dans cette démarche mais, d'un point de vue théorique, la littérature peine à trouver un consensus quant à une définition unifiée. Le BET est toutefois considéré comme un concept à part entière (Dagenais-Desmarais et Savoie, 2012), distinct de celui de santé mentale. Récemment, plusieurs auteurs considèrent qu'une mesure se focalisant uniquement sur les aspects positifs semble la plus pertinente pour apprécier les états de bien-être dans le contexte professionnel (Abaidi-Ben Nasr, 2015 ; Abord de Chatillon et Richard, 2015 ; Biétry et Creusier, 2013 ; Dasgenais-Desmarais et Savoie, 2012). En effet, les principales propositions antérieures de modèles pour la mesure du BET (Cotton et Hart, 2003 ; Daniels, 2000 ; Warr, 1990) s'appuient sur une combinaison simultanée de composantes négatives et positives, constituant finalement 
une mesure de la santé mentale au travail et non du BET tel qu'envisagé dans les recherches les plus récentes (ces concepts n'étant donc pas interchangeables).

Ainsi, à notre connaissance, peu de modèles ayant fait l'objet d'une validation empirique se consacrent réellement à la mesure du BET. Le modèle de Dagenais-Desmarais et Savoie (2012) élaboré à partir d'une méthodologie inductive, est constitué de deux axes : la sphère de référence (individuelle, relationnelle ou organisationnelle) et la directionnalité (projective ou introjective). Pour tenir compte de la sensibilité au contexte culturel (Christopher, 1999), Biétry et Creusier (2013) ont pour leur part développé un modèle adapté à la culture française en s'appuyant sur les travaux de Dagenais-Desmarais et Savoie (2012). Ce modèle repose sur une échelle à quatre dimensions : les relations avec les collègues, le rapport au management, le rapport au temps (stabilité des horaires, temps passé au travail, articulation vie personnelle / vie professionnelle) et l'environnement physique de travail. Il a permis aux auteurs de proposer une définition du concept du BET que nous retenons dans cet article : "un état psychologique résultant d'un rapport positif aux autres, à soi, aux temps et à l'environnement physique du travail ».

Cette approche par le BET est également constructive pour l'entreprise (dans sa démarche visà-vis du salarié) car elle permet, d'une part, de parler de manière positive de la santé pouvant constituer un point d'ancrage à une collaboration renforcée entre préventeur et manager (Grosjean, 2005) et, d'autre part, d'influer sur les principaux facteurs contribuant au bien-être professionnel tels que le maintien de la motivation au travail, la fidélisation de la main d'œuvre, la réponse à des contraintes légales et la lutte contre l'absentéisme. En conséquence, les bénéfices attendus par l'entreprise peuvent être nombreux : une meilleure efficacité individuelle (Lachmann, Larose et Penicaud, 2010 ; Wright et al., 2002), une meilleure qualité de travail (Lyubomirsky, Sheldon et Schkade, 2005), une productivité organisationnelle accrue (Harter, Schmidt et Hayes, 2002) et des comportements prosociaux plus nombreux (DagenaisDesmarais, 2010 ; Lee et Allen, 2002 ; Podsakoff et al., 2000).

Malgré l'émergence de cette approche positive, la plupart des études sur les TN se sont focalisées sur leurs aspects négatifs comme le stress technologique et ses répercussions sur les individus et l'organisation. Nous présentons ainsi dans les sections suivantes les études sur ces aspects négatifs pour ensuite synthétiser les recherches, moins nombreuses, sur l'influence des technologies nomades sur le BET.

\subsection{Les influences négatives des technologies nomades : le stress technologique ou technostress}

De nombreuses études font état d'une dégradation des conditions de travail depuis leur introduction. Cette détérioration qui diminue le BET se produit essentiellement à travers trois types de facteurs :

- la modification des liens hiérarchiques (Cézard et Hamon-Cholet, 1999; PeyratGuillard et Samier, 2004 ; Venin, 2013) ;

- la modification du contenu, de l'intensité et du rythme de travail (Bretesché, Corbière et Geffroy, 2012 ; Chevalet et Moatty, 2012 ; Greenan et al., 2012 ; Gupta, Li et Sharda, 2013 ; Isaac, Campoy et Kalika, 2007 ; Klein et Long, 2013 ; Peyrat-Guillard et Samier, 2004 ; Venin, 2013);

- la disparition de la frontière entre vie privée et vie professionnelle (Benedetto-Meyer et Klein, 2012 ; Cucchi, 2014 ; Venin, 2013).

Cette diminution du BET est assimilée au concept de stress technologique ou technostress. Celui-ci est une conséquence de plusieurs effets des TN sur les individus (effets appelés 
créateurs de stress) : une surcharge de travail accrue (Venin, 2013) et renforcée par le besoin de maitrise de la technologie (techno-overload), l'invasion de la technologie dans la sphère privée (techno-invasion), la complexité de la technologie (techno-complexity), l'insécurité de l'emploi liée à la crainte de non-adaptation au changement technologique (techno-insecurity) et l'incertitude liée aux changements rapides de technologies dans l'organisation (technouncertainty).

Sur le plan individuel, le manque de compétences sur la technologie et sa complexité favorisent fortement le stress technologique. Hudiburg et Necessary (1996) montrent ainsi que les individus souffrant le plus de technostress sont ceux les moins bien formés à l'usage de la technologie et les moins efficaces dans son utilisation. Rosen et Weil (2000) constatent alors que l'utilisation des TIC plus complexes est faite par les managers les moins technostressés. En effet, la formation individuelle aux technologies nouvellement mises en place est un modérateur important du stress technologique (Shu, Tu et Wang, 2011). Le stress lié à l'utilisation d'une technologie provient souvent du manque de congruence entre l'individu et son environnement technologique exigeant des compétences qu'il n'a pas (Al-Fudail et Mellar, 2008). À compétences constantes, en revanche, l'amélioration de la technologie elle-même rend l'adaptation des individus plus facile et réduit leur stress technologique (Ennis, 2005). Par ailleurs, le degré de dépendance à la technologie dans le travail quotidien augmente l'intensité du technostress des individus fortement dépendants (Shu, Tu et Wang, 2011).

En plus du stress lié à la complexité de la technologie et à l'incertitude qu'elle génère quant au maintien de son emploi, les interruptions fréquentes engendrées par l'utilisation des TN et la porosité entre la vie personnelle et la vie professionnelle sont parmi les facteurs les plus générateurs de stress. Par exemple, Wang, Shu et Tu (2008) documentent une relation positive entre technostress et surcharge de rôles, de même qu'avec les conflits de rôles. Les TIC contribuent à la multiplication des rôles perçus et subis par l'individu dans son travail qui doit faire face à davantage de demandes parfois incompatibles entre elles et génératrices de stress. La surcharge de travail (techno-overload) peut donc se traduire par une surcharge de rôles.

Enfin, la présence de stress technologique chez les individus soutient l'idée que les TIC, et les $\mathrm{TN}$ en particulier, sont davantage perçues comme une nouvelle exigence professionnelle pour les individus stressés. Toutefois, la question des TIC en tant que ressource pour l'individu, et non seulement en tant qu'exigence, est envisagée dans l'étude de Travaglianti (2011). Il montre par exemple que les téléphones intelligents sont au contraire davantage considérés (en moyenne) comme une ressource que comme une exigence nouvelle, mais que ces derniers augmentent les difficultés à concilier vie privée et vie professionnelle. L'auteur trouve également que ces téléphones expliquent en partie le burnout des individus les percevant comme une contrainte, notamment par la difficulté qu'ils ont à se détacher du travail.

Sur le plan organisationnel, les effets du technostress sont également visibles. En effet, si les conséquences du technostress affectent d'abord les individus, elles influencent aussi le (bon) fonctionnement de l'organisation. Plusieurs études se sont ainsi intéressées à l'impact du stress technologique sur la productivité du travail. Ayyagari (2007) montre notamment que le stress technologique engendre une baisse de productivité et une hausse des dépenses de santé pour les employés. Il relie les caractéristiques de la technologie (facilité d'usage, caractère intrusif, rythme d'évolution) aux éléments caractéristiques du technostress : surcharge de travail, ambiguïté de rôle, invasion de la vie privée, conflit travail-famille et insécurité de l'emploi. Hung, Chang et Lin (2011) mettent en évidence un double effet des créateurs de technostress (techno-overload, techno-invasion, accessibilité) sur la productivité d'employés utilisant des téléphones mobiles quotidiennement. Ils trouvent que si ces créateurs de stress favorisent 
directement et significativement la productivité des employés, l'augmentation conjointe du stress a un effet indirect négatif et significatif sur cette productivité.

Suivant l'intensité avec laquelle les créateurs de stress se manifestent, la résultante en termes de productivité est donc variable. Un juste équilibre semble devoir être trouvé sur le plan organisationnel lors de la mise en place des TN. Par exemple, toujours selon cette dernière étude, des inhibiteurs de stress (comme la mise en place d'entrainements à la gestion du stress, l'accroissement de la décentralisation et l'adaptation des récompenses individuelles) améliorent la productivité, et contrecarrent les effets négatifs du technostress. Tu, Wang et Shu (2005) montrent dans cette logique, sur un échantillon d'employés chinois, que l'invasion technologique et l'insécurité de l'emploi engendrées par la mise en place des TIC génèrent une productivité négative alors que la surcharge de travail permise par les TIC l'améliore.

Toujours sur le plan organisationnel, Peyrat-Guillard et Samier (2004) observent que des tensions entre supérieurs hiérarchiques et subordonnées peuvent naitre du fait de la pression accrue exercée sur ces derniers par le biais des TIC (notamment en termes de respect des délais, de satisfaction de la demande, de réduction des aléas et de réactivité). La pression de produire, associée à un manque de formation et d'entrainement, ou à un changement rapide de technologie, contribue en effet à l'augmentation du technostress chez les employés (Poole et Denny, 2001), générateur de tension avec les supérieurs. Pour limiter cet effet, un accompagnement des employés lors de l'introduction de la technologie est nécessaire pour modérer le technostress. Wang, Shu et Tu (2008) confirment que lorsque les individus perçoivent un support organisationnel pour gérer la technologie, la relation entre technostress et conflit de rôle devient négative.

Parallèlement à ces études mettant l'accent sur les conséquences négatives de l'introduction des $\mathrm{TN}$, d'autres recherches ont commencé à envisager les aspects positifs et leur apport au bienêtre des individus au travail. La section suivante présente les résultats de ces premières recherches puis synthétise l'ensemble de cette littérature sur les effets des TN.

\subsection{Les influences positives des technologies nomades : le bien-être technologique}

Malgré les impacts négatifs des TN, plusieurs études ont montré qu'elles pouvaient également améliorer le bien-être des salariés. Cette amélioration passe à la fois par une organisation individuelle plus efficace et plus flexible mais aussi par une organisation collective du travail offrant davantage de participation et de dialogue, ainsi qu'une décentralisation de la décision favorisant l'autonomie.

Sur le plan individuel, l'utilisation des TN fournit les moyens d'améliorer l'efficacité du travail par un accès rapide à une information pertinente. Les TN permettent donc de réduire la pression temporelle sur les autres tâches, à condition que ce gain de temps soit partagé équitablement entre le salarié et l'entreprise. De plus, les TN sont des outils de mise à disposition de l'information mais surtout de structuration de celle-ci. Les temps de traitement de l'information sont donc raccourcis, et permettent à l'individu de prendre des décisions plus pertinentes. Venin (2013) met par exemple en évidence la satisfaction des salariés ayant pour origine une meilleure efficacité perçue dans leur travail grâce à l'utilisation de téléphones intelligents.

Par ailleurs, une des caractéristiques principales des TN est d'étendre le temps et l'espace de travail. Pour les métiers concernés par un usage important de ces technologies (notamment les cadres), la connexion permanente aux outils de travail et à l'information donne à l'individu plus de flexibilité. Cette dernière, plus marquée en ce qui concerne l'organisation des tâches est à la fois temporelle (car les outils basés sur ces technologies favorisent le travail asynchrone) et géographique (le lieu virtuel de travail peut se substituer au lieu physique). Le travail devient 
« ubiquitaire» (Besseyre des Horts et Isaac, 2006) car il peut s'effectuer n'importe où et n'importe quand, grâce à la mobilité des technologies. Si cette flexibilité peut à terme se transformer en contrainte, le travailleur bénéficiant de l'usage de TN peut ainsi adapter son emploi du temps professionnel à son emploi du temps privé. Bien évidemment, cette flexibilité n'est possible que pour les métiers qui ne nécessitent pas d'effectuer les tâches uniquement sur le lieu de travail. Les rapports de Klein et Long (2013) et du GRePS-Apec (2011) détaillent et documentent ces effets.

Sur le plan organisationnel, plusieurs études rapportent que l'usage des TN est associé à la présence d'instances et de mécanismes de participation au sein des entreprises. Peyrat-Guillard et Samier (2004) montrent par exemple que les TIC (dont font partie les TN) sont associées à une présence plus importante de dispositifs de participation et de groupes de travail dans l'entreprise. L'usage des TIC permet à ces dispositifs d'accroître le dialogue dans l'entreprise et la participation aux décisions. Enfin, l'autonomie des salariés est également renforcée par l'usage de ces technologies : l'organisation du travail par objectif (Peyrat-Guillard et Samier, 2004), un intranet et des outils collaboratifs rendent l'individu moins dépendant de la hiérarchie dans son travail quotidien et décentralisent la prise de décision. Les problèmes mineurs peuvent être pris en charge par le salarié lui-même tandis que les décisions sont prises de façon asynchrone et distante (hors cadre formel des réunions, des comités de direction, etc.).

Ces différents effets des technologies nomades sont donc susceptibles d'améliorer le bien-être au travail des salariés. L'existence chez ces derniers d'un bien-être que l'on pourrait alors qualifier de "technologique» ne doit toutefois pas occulter les effets négatifs de ces technologies, notamment par l'augmentation du stress qu'elles peuvent induire. Les concepts de bien-être et de stress technologique n'étant pas strictement les négatifs l'un de l'autre, il semble important d'envisager les deux conjointement dans la mesure où un état de bien-être lié à l'utilisation des TN n'est pas incompatible avec un état de stress lié à l'utilisation de ces mêmes TN.

Nous envisageons dans cet article qu'une augmentation du stress technologique ou technostress peut toutefois cohabiter avec un état de BET en fonction de son intensité. N'étant pas des concepts équivalents et mutuellement exclusifs, le technostress et le bien-être technologique ne sont pas incompatibles, alors que peu d'études les envisagent conjointement. En fonction de l'intensité avec laquelle chaque créateur de stress se manifeste chez les individus, le BET se dégrade généralement, mais s'améliore parfois sous certaines conditions que nous étudions dans cet article. Un soutien organisationnel (support technique, accompagnement, etc.) mis en place lors de l'introduction des TN peut par exemple réduire l'influence du technostress et garantir un niveau constant de BET. Ragu-Nathan et al. (2008) considèrent même que le niveau de satisfaction au travail peut compenser l'effet du stress technologique, au même titre que le soutien organisationnel à l'introduction et à l'usage des TN. C'est cette relation entre technostress et BET que nous cherchons à caractériser lors de la mise en place de TN. Le tableau 1 résume les facteurs d'impact des TN sur les individus et l'organisation, à la fois en termes de BET mais aussi de technostress, sur la base de la littérature présentée ci-avant. 
Tableau 1. Les TN, génératrices de bien-être et/ou de stress au travail

\begin{tabular}{|c|c|c|}
\hline & Sources de BET (techno-bien-être) & Sources de stress (technostress) \\
\hline $\begin{array}{l}\text { Impacts des TN } \\
\text { sur le plan } \\
\text { individuel }\end{array}$ & $\begin{array}{l}\text { Réduction de la pression } \\
\text { temporelle sur les tâches } \\
\text { quotidiennes et meilleure efficacité } \\
\text { perçue par l'individu (Venin, 2013) } \\
\text { en raison : } \\
\quad \text { - d'un accès rapide aux } \\
\quad \text { informations pertinentes ; } \\
\quad \text { - d'une structuration de ces } \\
\quad \text { informations réduisant les } \\
\quad \text { temps de traitement et } \\
\quad \text { facilitant la prise de } \\
\quad \text { décision } \\
\text { Flexibilité spatiale et temporelle } \\
\text { dans l'organisation du travail } \\
\text { (« travail ubiquitaire », Besseyre } \\
\text { des Horts et Isaac, 2006), } \\
\text { permettant une meilleure } \\
\text { articulation des vies privée et } \\
\text { professionnelle (GRePS-Apec, } \\
\text { 2011 ; Klein et Long, 2013) }\end{array}$ & $\begin{array}{l}\text { Complexité des TN qui exigent de } \\
\text { nouvelles compétences et } \\
\text { l'adaptation des individus : le } \\
\text { manque de compétences augmente } \\
\text { le stress et la peur de perdre son } \\
\text { emploi, d'autant plus si le travail } \\
\text { dépend des technologies (Al-Fudail } \\
\text { et Mellar, 2008; Ennis, 2005; } \\
\text { Hudiburg et Necessary, 1996; } \\
\text { Rosen et Weil, 2000; Shu, Tu et } \\
\text { Wang, 2011) } \\
\text { Interruptions fréquentes du } \\
\text { travail quotidien, concentration } \\
\text { plus difficile } \\
\text { Porosité entre vies privée et } \\
\text { professionnelle, surcharge et } \\
\text { conflits de rôle (Travaglianti, } \\
2011 \text {; Wang, Shu et Tu, 2008) }\end{array}$ \\
\hline $\begin{array}{l}\text { Impacts des TN } \\
\text { sur le plan } \\
\text { organisationnel }\end{array}$ & $\begin{array}{l}\text { Participation accrue aux } \\
\text { décisions et meilleur dialogue } \\
\text { (présence plus importante de } \\
\text { dispositifs de participation et de } \\
\text { groupes de travail, Peyrat-Guillard } \\
\text { et Samier, 2004) } \\
\text { Meilleure autonomie quotidienne } \\
\text { et décentralisation des décisions } \\
\text { (Peyrat-Guillard et Samier, 2004) }\end{array}$ & $\begin{array}{l}\text { Surcharge de travail et ambiguïté } \\
\text { de rôle impliquant un stress accru } \\
\text { et une baisse de la productivité } \\
\text { (Ayyagari, 2007; Hung, Chang et } \\
\text { Lin, 2011) } \\
\text { Tensions entre supérieurs } \\
\text { hiérarchiques et subordonnés } \\
\text { liées à une pression accrue via les } \\
\text { TN (Peyrat-Guillard et Samier, } \\
2004 \text {; Poole et Denny, 2001) }\end{array}$ \\
\hline
\end{tabular}

Au-delà des conséquences du technostress sur les individus, la relation entre celui-ci et le BET n'est pas immédiate et reste peu étudiée. Par exemple, Korunka, Zauchner et Weiss (1997) évaluent les effets sur le stress et la satisfaction au travail à la suite de la mise en place d'une nouvelle TIC sur deux ans dans plusieurs organisations. Ils montrent que cette introduction augmente le stress perçu à court terme mais pas l'insatisfaction. Il semble donc qu'il y ait un découplage entre la satisfaction au travail et le technostress. Pour envisager ce découplage, nous utilisons le modèle job demands-resources (JD-R) révisé (Schaufeli et Bakker, 2004 ; Schaufeli et Taris, 2014) qui permet de faire le lien entre stress technologique et bien-être technologique par le biais des TN, à la fois exigence professionnelle (contrainte) et ressource pour l'individu. 


\subsection{Modèle JD-R «exigences professionnelles-ressources" adapté aux effets des technologies nomades}

Demerouti et al. (2001) caractérisent initialement le développement de l'épuisement professionnel (burnout) par les relations entre les exigences professionnelles du travail et les ressources dont l'individu dispose pour y répondre. Plus l'écart entre les exigences formulées ou perçues et les ressources de l'individu est fort, plus le risque d'épuisement professionnel s'accroit. Les notions d'exigences et de ressources sont définies volontairement de façon assez large. Les exigences sont les caractéristiques physiques, psychologiques, sociales et organisationnelles de l'emploi qui nécessitent des efforts physiques et mentaux soutenus, et qui représentent donc un coût physique ou psychologique pour l'individu (Bakker et Demerouti, 2007 ; Demerouti et al., 2001). Les ressources sont a contrario les moyens physiques, psychologiques, sociaux et organisationnels (tels que des objets, les conditions de travail, les énergies disponibles et les caractéristiques personnelles) permettant à l'individu de réduire le coût psychologique ou physiologique des exigences de son emploi (Bakker et Demerouti, 2007 ; Demerouti et Bakker, 2011 ; Hakanen, Schaufeli et Ahola, 2008 ; Meijman et Mulder, 1998). En plus de répondre aux exigences de l'emploi, les ressources peuvent être utilisées pour en développer de nouvelles (Diener et Fujita, 1995). En fonction de la disponibilité des ressources, l'individu va pouvoir réagir plus ou moins rapidement, et plus ou moins efficacement, aux créateurs de stress issus des exigences de l'emploi. En cas de décalage trop important entre les ressources disponibles et les exigences perçues, et dans la mesure où ce décalage persiste dans le temps, le stress induit peut contribuer à dégrader l'état de santé de l'individu (Bakker et al., 2003 ; Demerouti et al., 2009). La figure 1 présente le modèle original de Demerouti et al. (2001).

\section{Figure 1. Modèle JD-R de Demerouti et al. (2001)}

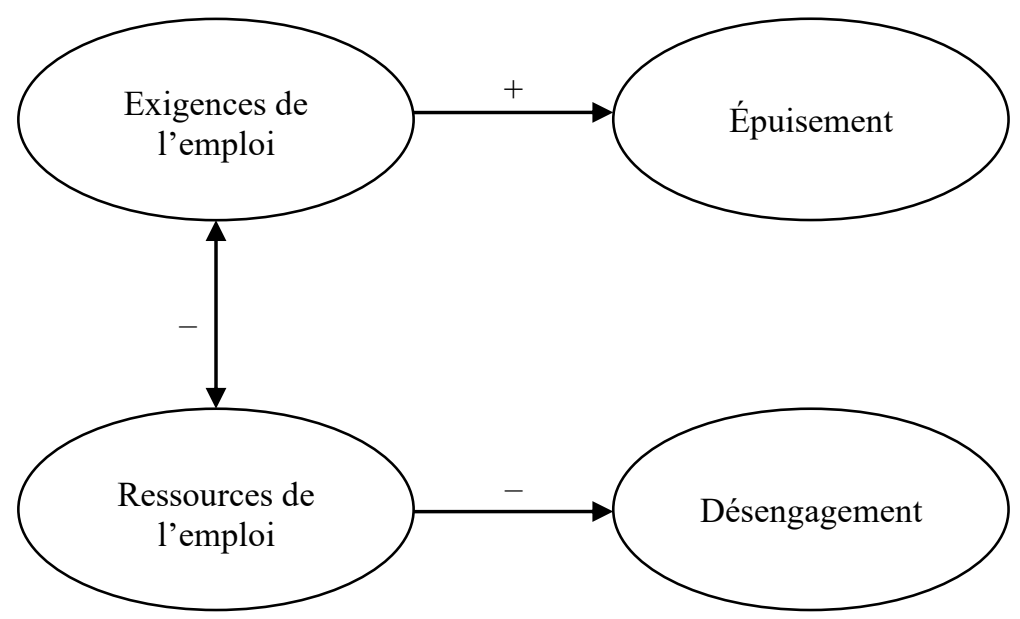

Ce modèle d'explication de l'épuisement professionnel reste toutefois focalisé uniquement sur les aspects négatifs issus du déséquilibre entre exigences et ressources. Or, plusieurs aspects positifs peuvent conjointement émerger de cette relation, ayant engendré plusieurs adaptations du modèle initial dans la littérature. C'est ainsi qu'une première version révisée du modèle JDR par Schaufeli et Bakker (2004) a permis d'étendre l'analyse de l'épuisement professionnel à l'engagement au travail, donnant par conséquent une modalité d'analyse positive au modèle initial (Schaufeli et Taris, 2014) ${ }^{3}$, modalité qui sera ensuite développée dans les recherches suivantes. Ce modèle révisé présente donc l'avantage de pouvoir étudier simultanément des effets, positifs comme négatifs, pouvant refléter des situations de mal-être et de bien-être

\footnotetext{
${ }^{3}$ « Le modèle JD-R révisé a non seulement cherché à expliquer un état psychologique négatif (c. -à-d. l'épuisement professionnel) mais aussi son homologue positif (l'engagement au travail)» (Schaufeli et Taris, 2014, p. 46).
} 
conjointes chez des individus au travail. Deux processus principaux sont en effet à l'œuvre dans la version révisée du modèle JD-R présenté en figure 2 (Schaufeli et Taris, 2014) :

(1) Un processus négatif, faisant état de la dégradation du bien-être des individus lorsque les exigences du métier sont durablement trop fortes par rapport aux ressources disponibles. Dans le cas extrême, un épuisement professionnel peut émerger et induire des effets négatifs importants sur la santé de l'individu.

(2) Un processus motivationnel, donc positif, issu des ressources, pouvant mener au bien-être, à l'engagement au travail, et in fine à une performance accrue. En effet, Schaufeli et Taris (2014, p. 47) précisent que «les ressources jouent un rôle motivationnel extrinsèque parce qu'elles engendrent la volonté de faire un effort compensatoire réduisant les exigences du travail et favorisant l'atteinte des objectifs ». Ils rappellent également le rôle intrinsèque des ressources dans la motivation au travail car elles permettent de «satisfaire les besoins humains basiques d'autonomie, de connexion et de compétence ».

Figure 2. Modèle JD-R révisé (Schaufeli et Taris, 2014, p. 46)

Processus de dégradation de la santé

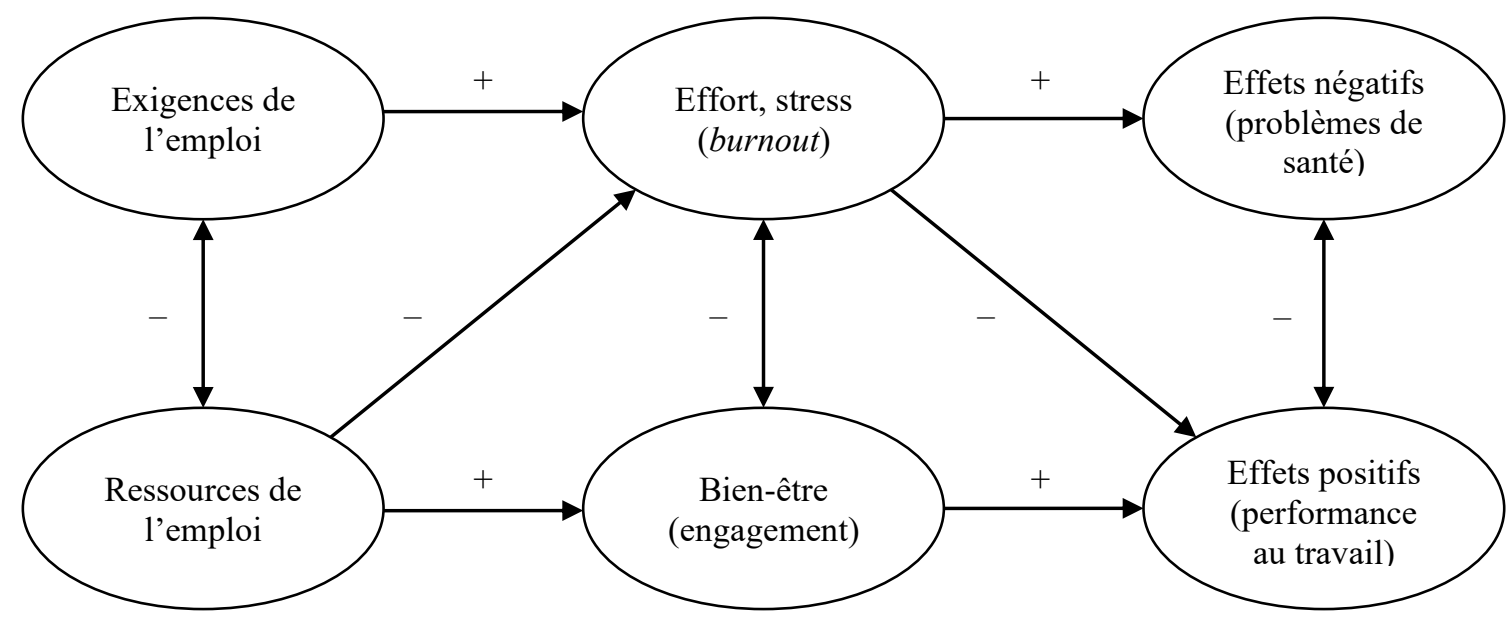

Processus motivationnel

Note : les signes $(-)$ indiquent une relation inverse entre les concepts, les signes $(+)$ traduisent une relation de même sens.

Dans cette version révisée du modèle JD-R, les états mentaux des individus issus du décalage entre exigences professionnelles et ressources sont multidimensionnels, pouvant être négatifs (cas extrême du burnout) comme positifs (engagement au travail, bien-être, motivation, etc.).

Ce modèle JD-R étant heuristique par nature, il peut être adapté à de nombreux contextes (Schaufeli et Taris, 2014) dès lors que les caractéristiques du travail sont susceptibles d'influencer la santé, le bien-être et la motivation au travail. Le contexte des TN semble particulièrement pertinent à analyser dans ce cadre puisque la littérature étudiant les effets de l'introduction des TN (cf. tableau 1) montre que celle-ci produit à la fois des effets positifs et négatifs sur les individus. Pour mieux comprendre l'origine de ces effets, une analyse montrant les décalages possibles entre exigences et ressources liées à l'introduction des TN nous semble prometteuse. En effet, l'introduction des TN exige de nouvelles compétences et efforts de la part des individus (Al-Fudail et Mellar, 2008 ; Ennis, 2005 ; Hudiburg et Necessary, 1996 ; Rosen et Weil, 2000 ; Shu, Tu et Wang, 2011) qui ne disposent pas toujours des ressources adéquates pour faire face d'une part, à l'augmentation de la charge de travail induite par ces 
technologies (Ayyagari, 2007 ; Hung, Chang et Lin, 2011) et, d'autre part, à la maîtrise des technologies elles-mêmes. Dans le même temps, les TN, lorsqu'elles sont maîtrisées, constituent une ressource mobilisable pour réduire l'écart avec les exigences professionnelles ; elles engendrent notamment autonomie décisionnelle, flexibilité et réduction de la pression temporelle (GRePS-Apec, 2011; Klein et Long, 2013; Peyrat-Guillard et Samier, 2004 ; Venin, 2013). Les modèles issus des travaux de Schaufeli et Bakker (2004) qui révisent la version initiale de Demerouti et al. (2001) permettent alors d'envisager le découplage entre le caractère contraignant des TN (exigences professionnelles) pouvant conduire au technostress et les ressources supplémentaires qu'elles apportent, susceptibles d'améliorer le BET. Nous proposons donc un cadre d'analyse issu du modèle JD-R révisé (Schaufeli et Bakker, 2004 ; Schaufeli et Taris, 2014) mais adapté aux situations de travail mobilisant des TN. En effet, nous souhaitons (1) identifier quelles sont les exigences et ressources spécifiques à l'introduction des $\mathrm{TN},(2)$ comprendre comment leur combinaison peut engendrer conjointement des situations de stress technologique et de bien-être technologique, et analyser l'influence de ces situations sur la performance et la santé au travail en prenant comme unité d'analyse l'individu. Nous adaptons ainsi le modèle présenté en figure 2 au cas des TN en remplaçant les concepts de bienêtre (engagement) et de stress (burnout) par les concepts de bien-être technologique et de technostress puisque nous nous focalisons sur les exigences et les ressources procurées par les TN. La figure 3 présente ce cadre d'analyse que nous utiliserons dans la suite de l'article.

Figure 3. Modèle JD-R révisé appliqué à l'introduction des TN (adapté de Schaufeli et Taris, 2014)

Processus de dégradation de la santé

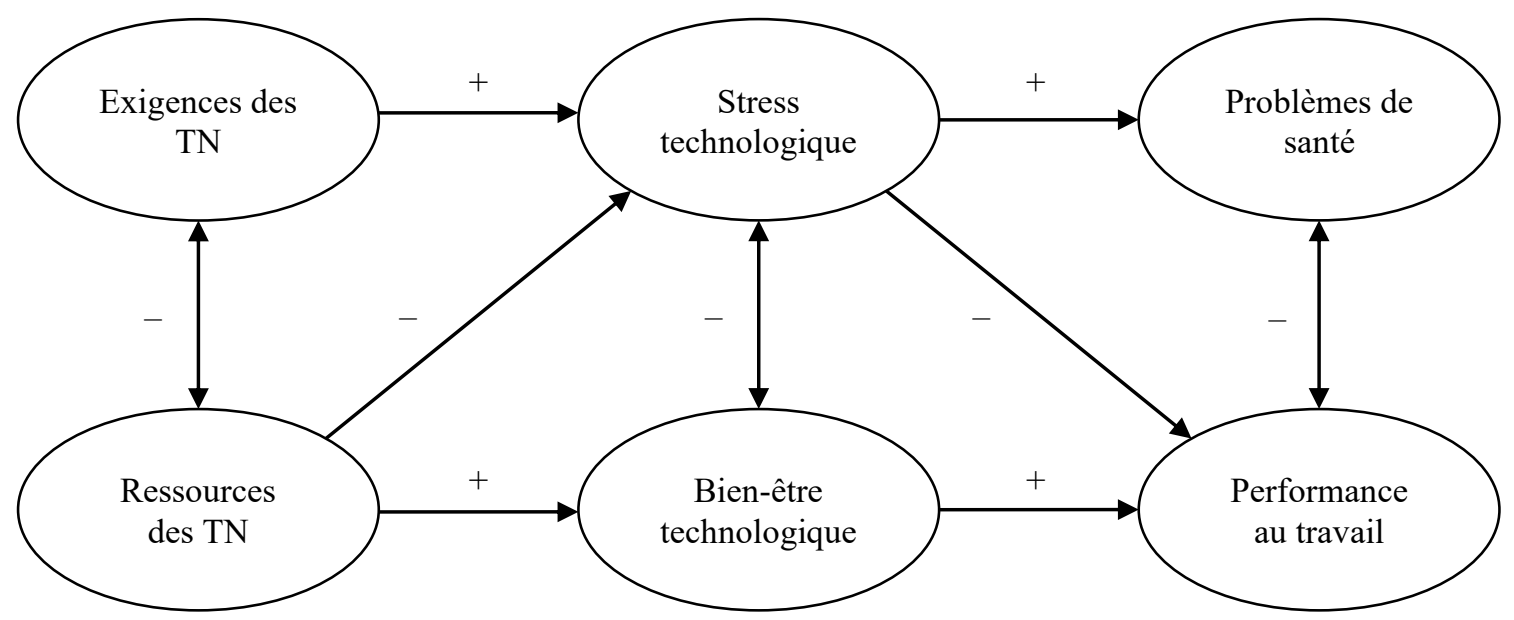

Processus motivationnel

Note : les signes (-) indiquent une relation inverse entre les concepts, les signes $(+)$ traduisent une relation de même sens.

\section{Méthode : recueil et analyse de données qualitatives}

Avant de pouvoir éventuellement tester quantitativement le modèle ci-dessus dans de futures recherches, il semble nécessaire de déterminer qualitativement quelles sont les exigences et les ressources spécifiques aux TN, telles qu'elles sont perçues par les individus au travail. Une fois ces perceptions mises en lumière, il sera alors possible de comprendre comment la combinaison de ces exigences-ressources peut engendrer conjointement des situations de stress 
technologique et de bien-être technologique au niveau individuel. Pour ce dernier point, le recours à une méthodologie qualitative semble plus adapté. En effet, selon Firestone (1987), si les données quantitatives présentent l'avantage de fournir des résultats plus généralisables, l'approche qualitative quant à elle permet de riches descriptions et propose une vision moins abstraite de la situation, a fortiori dans un contexte où les relations entre concepts ne sont pas bien établies comme pour le lien entre TN, stress et bien-être. En outre, une méthode basée sur le recueil et l'analyse de données qualitatives est pertinente pour répondre à une question de type « comment » (Yin, 2017) en privilégiant la profondeur de la description à la recherche de régularités.

Afin de répondre à nos questions de recherche, nous avons eu l'opportunité d'étudier les effets de l'introduction de TN au sein du groupe La Poste, d'interroger et d'observer les personnes concernées à différents niveaux hiérarchiques. Nous présentons dans les sections suivantes le contexte empirique de l'étude, le mode de recueil des données qualitatives ainsi que la démarche d'analyse.

\subsection{Contexte empirique de l'étude}

À partir de 2012, les équipes commerciales du groupe La Poste ont fait l'objet d'un déploiement de TN (ordinateurs convertibles en tablettes et mobiles multifonctions) afin de faciliter les échanges et la diffusion d'informations au sein de l'entreprise et avec les clients. Les managers, mais également les commerciaux, sont concernés par cette action. Les conséquences de ce déploiement sur la vie au travail, et notamment sur le BET, font l'objet d'une attention particulière par le groupe La Poste, signataire par ailleurs d'un document en faveur du BET et du droit universel à la santé auprès de l'Observatoire social international (OSI) en mai 2010. Cette démarche fait également écho au rapport Kaspar, remis en septembre 2012 à la demande de Jean-Paul Bailly (alors PDG du groupe La Poste), et dont l'objectif principal était d'ouvrir des pistes de réflexions et d'actions permettant à La Poste d'entreprendre les bases d'une refondation des pratiques de gestion, managériales et sociales, dans une optique d'amélioration du BET.

\subsection{Collecte des données qualitatives}

Le groupe La Poste a fait l'objet d'une étude de cas unique dans ce contexte propice à l'analyse de l'influence des TN sur le BET. Elle a été menée au sein des équipes commerciales du grand quart Sud-Est de la France (dans 6 entités couvrant 18 départements) et s'est déroulée sur une période de 6 mois (d'avril à septembre 2014). Au cours de cette période, 34 entretiens semidirectifs (en face-à-face ou par téléphone) ont été réalisés sur une population cible de 133 personnes. La durée moyenne des entretiens effectués est de 55 minutes (avec une étendue allant de $36 \mathrm{~min}$ à $1 \mathrm{~h} 42 \mathrm{~min}$ ). Le nombre d'entretiens réalisés par entité varie de 5 à 8 assurant une couverture relativement homogène des différents sites. Notre échantillon est composé de trois strates hiérarchiques : les fonctions de direction ( 7 entretiens dont un avec la direction opérationnelle de la région géographique considérée), les fonctions d'encadrement (12 entretiens) et les fonctions commerciales (15 entretiens). L'échantillon est composé de $42 \%$ de femmes et $58 \%$ d'hommes. Les âges des personnes interviewées sont compris entre 24 et 55 ans, avec une moyenne à 43,5 ans. Les personnes interrogées ont une ancienneté moyenne au sein de La Poste de 16,8 années, sachant que $40 \%$ d'entre-elles n'ont jamais travaillé dans d'autres organisations. Après 34 entretiens, peu d'informations nouvelles étaient amenées malgré la diversité des profils interviewés mettant ainsi en exergue un effet de saturation. Nous avons donc jugé inutile de poursuivre la collecte de données. Les thèmes 
suivants ont été abordés au cours des entretiens : les caractéristiques du métier, l'usage et les conditions d'utilisation des TN déployées, la vision de la vie au travail par l'interviewé, et l'influence de l'arrivée des TN sur l'organisation et la santé au travail. Le guide d'entretien complet est présenté en annexe 1 avec les objectifs associés aux questions.

En plus de ces entretiens, plusieurs jours d'observation flottante (cf. tableau 2) sur trois des sites étudiés (Montpellier, Valence et Nice) ont contribué à immerger l'un des chercheurs au sein des équipes commerciales. Ce genre d'observation dite «flottante », c'est-à-dire qui n'a pas un caractère systématique (comme peuvent le nécessiter les observations participantes ou non-participantes), permet de mieux s'imprégner du terrain (Olivier de Sardan, 1995). Lors de sa présence dans les locaux professionnels des personnes interviewées, le chercheur peut au fil de l'eau saisir des contextes, des situations et des ambiances particulières lui permettant une meilleure compréhension du phénomène étudié. Cette immersion ponctuelle a ainsi donné lieu au partage de moments de convivialité (discussions informelles, pauses café, pauses déjeuner) et à de nombreux échanges avec les acteurs de terrain.

À ces jours d'observation flottante s'ajoute également la participation d'un des chercheurs à deux événements organisés par le groupe La Poste concernant ses équipes commerciales : un forum informatique où l'ensemble des applications informatiques dédiées au personnel des équipes commerciales du courrier était présenté et un forum du management portant sur les pratiques managériales et leurs influences potentielles sur la santé mentale au travail. Enfin, trois restitutions des conclusions de cette recherche ont été réalisées auprès de la directrice opérationnelle du grand quart Sud-Est, puis de certaines directions et équipes commerciales, ce qui a permis de confirmer leur validité. Le tableau 2 synthétise l'étendue du matériau empirique sur lequel repose cette recherche.

\section{Tableau 2. Présentation synthétique des sources des données collectées}

\begin{tabular}{rr}
\hline Entretiens semi-directifs & $\mathbf{3 4}$ \\
\hline Avec la direction opérationnelle & 1 \\
Avec la direction commerciale & 6 \\
Avec les managers & 12 \\
Avec les commerciaux & 15 \\
\hline Jours d'observation flottante & $\mathbf{8}$ \\
\hline Site de Montpellier & 5 \\
Site de Nice & 2 \\
Site de Valence & 1 \\
\hline Événements La Poste & $\mathbf{2}$ \\
\hline Restitutions avec les acteurs & $\mathbf{3}$
\end{tabular}

\subsection{Analyse des données collectées}

Les 34 entretiens réalisés représentent plus de 31 heures d'enregistrement. Lors du processus de transcription, nous nous sommes attachés à retranscrire le plus fidèlement possible et de manière exhaustive les propos des personnes interviewées. Cette étape, préalable à la phase d'analyse, nous a permis de nous imprégner du contenu des entretiens. À l'issue de cette dernière, le corpus obtenu est composé de 551 pages (police 11, interligne 1,15). L'analyse de 
corpus a été conduite à l'aide du logiciel Nvivo 11 afin de réaliser un codage thématique (Fallery et Rodhain, 2007). Les catégories inhérentes au codage ont été constituées a priori (Miles et Huberman, 2003), sur la base de la littérature précédente et enrichies au fil des entretiens. Le recours à un logiciel de traitement a essentiellement été guidé par le volume important de notre corpus de données qui nécessitait la mise en place d'une méthodologie de traitement nous permettant d'augmenter la vitesse d'analyse et facilitant l'accès à des éléments statistiques et au croisement de données (Wanlin, 2007). Ainsi, par le biais d'une interface ergonomique, Nvivo permet l'import, l'organisation et la gestion des données recueillies dans le cadre de l'enquête. Le logiciel facilite également la systématisation de la démarche mise en place pour l'analyse, ce qui constitue un atout considérable lorsque les données sont volumineuses. L'utilisation de Nvivo a permis un codage en deux étapes (Fallery et Rodhain, 2007) : une première étape de codage libre et manuelle conduit à décontextualiser le corpus en stockant, qualifiant et réorganisant les informations grâce à la création de catégories (nœuds) initiales non hiérarchisées ; une seconde étape de recontextualisation s'est ensuite traduite par le regroupement des différents nœuds en catégories plus vastes et hiérarchisées pour obtenir in fine « un tout intelligible et porteur de sens » (Fallery et Rodhain, 2007, p. 11). L'annexe 2 présente l'arbre de codage utilisé, avec des exemples de regroupements de nœuds pour l'analyse.

L'analyse des données a conduit à formuler, en discussion, des propositions de recherche et à enrichir le modèle JD-R applicable aux TN. Pour assurer la validité des résultats sur le plan scientifique, nous avons réalisé trois restitutions aux acteurs de terrain (auprès de la directrice opérationnelle puis de certaines directions et équipes commerciales). L'ensemble des acteurs a confirmé la pertinence des conclusions de l'étude qui synthétisent bien, selon eux, l'influence des TN dans leur quotidien.

\section{$4 \quad$ Résultats : une double influence des TN entre stress et bien-être}

L'objectif de ce travail est d'explorer l'influence de l'introduction de TN sur le bien-être ou le stress au travail au travers des usages qui en sont faits, tant par la ligne hiérarchique que par les utilisateurs. Pour cela, nous cherchons à évaluer si ces technologies sont perçues comme des exigences de travail ou des ressources supplémentaires (modèle JD-R) pouvant entrainer du stress et/ou du bien-être. Au cours des entretiens menés au sein des équipes commerciales de La Poste, la question du bien-être et du stress au travail à la suite de l'introduction des TN a été largement abordée. Les discussions autour de cette thématique ont été riches, y compris pendant les périodes d'observation flottante. Ces discussions ont été souvent illustrées par des situations de travail propres aux interlocuteurs, mettant en évidence leurs ressentis positifs comme négatifs vis-à-vis des TN dans leur travail quotidien. Nous proposons dans cette section une analyse des entretiens et des notes d'observation présentant les effets des TN sur l'organisation et l'individu, dans la perspective du modèle JD-R présenté en section 2. L'un des résultats principaux et original de cette recherche est la mise en lumière de certains couples exigencesressources qui agissent de façon simultanée sur le stress et le bien-être au travail des individus interrogés ${ }^{4}$.

\footnotetext{
${ }^{4}$ Les couples proposés émergent de l'analyse des entretiens et doivent être considérés comme des couples principaux et non-exhaustifs. D'autres relations entre exigences et ressources peuvent exister à la marge mais ne sont pas détaillées ici dans la mesure où elles n'ont pas massivement émergé du terrain.
} 


\subsection{Une augmentation de la charge de travail et de l'immédiateté compensée par une réactivité accrue}

\section{Exigence - Augmentation de la charge de travail}

Le déploiement des TN au sein des équipes commerciales de La Poste a modifié l'organisation du travail, la fréquence et le contenu des échanges en interne (communications horizontale et verticale) ou avec la clientèle. Ces outils sont totalement intégrés dans les processus organisationnels et jouent un rôle central dans le quotidien des interviewés. Ainsi, les changements organisationnels tournés vers un pilotage plus décentralisé nécessitent la production de nombreux tableaux de bord, comptes rendus ou autres remontées d'informations venant en plus des activités classiques et ayant une influence directe sur l'amplitude horaire du travail :

«Donc pour décentraliser effectivement les domaines de compétences, ils ont resserré, centralisé le pilotage, le suivi donc il y a un effet pernicieux et ça augmente les tâches de travail, ça augmente l'amplitude horaire. C'est-à-dire que par rapport à il y a un an ou deux mon amplitude horaire a augmenté de plus d'une heure. [...] Ça a énormément changé l'activité, la façon de gérer son temps, c'est devenu extrêmement chronophage. Pourquoi? Parce qu'on a accès à tout, parce qu'on est joignable partout. Inversement, on peut joindre tout le monde et envoyer des messages à tout le monde n'importe où, n'importe quand. Après, il faut se discipliner c'est évident, mais tous les gens ne sont pas disciplinés, donc ça a changé pas mal mon temps de travail. » (Manager 25)

Cette plus grande charge de travail liée au nomadisme s'exprime notamment par une profusion d'informations, une accumulation de sollicitations conduisant à des interruptions d'activités et est souvent synonyme de travail supplémentaire. Nous assistons ainsi à une intensification et une densification du travail liées à l'introduction des $\mathrm{TN}$, ces dernières engendrant une diminution des temps morts et une mutation profonde dans la gestion du temps :

«Le risque, c'est cette sollicitation permanente. Si on ne la maitrise pas, on se met en risque. [...] Ça fait monter le niveau d'exigence en termes de réactivité, il n'y a pas de doute là-dessus, plus d'engagement personnel, on a moins le temps de faire les choses, donc le fait d'avoir accès partout en permanence à notre outil de travail est un confort mais c'est vrai que c'est une source, j'imagine, de fatigue, de stress. » (Directeur commercial 13)

\section{Exigence - Immédiateté et pression de la réponse}

La facilité de communication offerte par les TN intensifie par ailleurs les échanges en favorisant ainsi l'immédiateté qui s'accompagne souvent d'un phénomène de " pression de la réponse » ressenti par les interlocuteurs :

«Il y a un effet pervers aussi qui est, qu'aujourd'hui, quand on vous envoie un mail, en général dans les dix minutes si vous n'avez pas répondu, il y a quelqu'un qui vient et qui dit "mais t'as pas vu mon mail? ". Donc c'est le côté un peu pervers. » (Manager 13)

«Je vous donne un exemple, j'ai une réunion de 14 h à 16 h, et puis j'ai un gros dossier à terminer, mais il y a un mail, quelque chose qui tombe, il faut s'en occuper tout de suite, parce qu'on bosse beaucoup dans l'urgence, l'urgence est devenue un mode de fonctionnement. » (Manager 5) 
«Maintenant, les gens ont une exigence en termes de réactivité qui devient délirante. C'est-à-dire, que sous prétexte que vous ne répondez pas à un mail, ça $m$ 'est arrivé deux ou trois fois, des clients qui me disent j'ai essayé de vous joindre toute la matinée. Ça a créé une espèce d'exigence en termes de réactivité qui, je trouve, est encore accentuée par les smartphones. » (Manager 28)

«On subit et alors après est-ce que c'est mieux, moins bien? Je pense que le stress va augmenter parce que l'ère de la communication est de plus en plus rapide. Les gens s'attendent à avoir une information très rapide. " (Manager 21)

Un premier groupe d'exigences professionnelles provenant directement de l'introduction des technologies nomades est mis en évidence dans ces verbatims : l'augmentation de la charge de travail et, par extension, des amplitudes horaires du travail ainsi que la pression de la réponse dans l'immédiateté des échanges (exigence de réactivité). Les termes utilisés par les personnes interrogées (en gras dans les verbatims) révèlent bien que ces exigences, si elles ne sont pas compensées par des ressources supplémentaires pour les individus, peuvent engendrer un stress important (p. ex., intensité folle, côté pervers, effet pernicieux, exigence délirante, urgence, fatigue). Elles illustrent bien le premier processus de notre modèle JD-R (figure 3).

\section{Ressource - Réactivité accrue par des échanges plus fluides}

L'accroissement des exigences du travail lié aux TN peut parfois donner aux membres des équipes commerciales la sensation d'être débordés mais, malgré cela, les TN restent fortement plébiscitées par les interviewés et ce pour plusieurs raisons. En premier lieu, la quasi-totalité des personnes interviewées louent l'accessibilité en termes d'utilisation des outils déployés (le téléphone intelligent, en particulier, étant un outil familier pour une grande majorité) et également leur fiabilité (peu de problèmes techniques recensés, associés à une efficacité reconnue du service informatique). Ainsi, les TN mises à disposition des équipes commerciales sont souvent reconnues comme fluidifiant les échanges. L'accessibilité aux mails permet notamment une gestion plus efficace de ces derniers (évitant les craintes liées à l'interrogation : «Je charge mes mails... tout rentre..., qu'est-ce que je vais trouver? », directeur commercial 31) et amène une bien meilleure réactivité, prépondérante dans le milieu commercial :

«En même temps, ça me permet aussi d'être plus réactive sur certaines problématiques. C'est très, très, très important, ça nous a permis aussi de signer pas mal de trucs. » (Commercial 3)

Les TN permettent donc aux individus de gagner du temps: les notions de réactivité, d'immédiateté, d'instantanéité dans les échanges, de rapidité d'accès à l'information et aux données, de possibilité de travailler en tous lieux et donc d'éviter des pertes de temps liées aux déplacements sont clairement évoquées :

«Je reste intimement persuadée que ça change et que ça facilite la vie, toujours sur cette notion de gain de temps. » (Manager 15)

«Par contre, effectivement, comme je suis à temps partiel, pour moi au contraire c'est un gain de temps. » (Commercial 19)

Tous ces points contribuent à une plus grande efficacité des équipes commerciales et à une amélioration de leur performance qui semble étroitement liée à ces dispositifs :

«La performance de l'entreprise ne se fait pas sans ça et je pense que si on n'est pas aujourd'hui dans ces médias-là, dans ces moyens de communication-là, on n'est pas dans le coup en termes de relation client. »(Directeur commercial 13) 
«Je trouve qu'on est beaucoup plus efficace et direct dans les échanges de mails et textos qu'en face à face. Il faut de l'échange en face à face mais souvent quand on va voir quelqu'un, ça prend des plombes. Là, tu envoies un texto « est-ce que tu es libre tel jour pour tel rendez-vous? ?. Il te répond "oui, non », effectivement, en 30 secondes c'est réglé. Donc il me semble qu'on est beaucoup plus efficace et réactif dans les échanges de textos et de mails que dans la relation directe. » (Commercial 11)

Enfin, même si, comme évoqué précédemment, une certaine forme de " pression de la réponse » peut être ressentie par certains utilisateurs, en contrepartie un grand nombre d'entre eux trouvent rassurant le fait de se savoir joignable, particulièrement pour ne pas manquer une affaire commerciale...

" [En déplacement] on a au moins la messagerie, qui nous permet d'être connecté avec nos équipes, avec nos clients, ça c'est important parce que du coup, on peut réagir rapidement s'il y a un problème donc juste pour ça c'est rassurant, c'est ce côté-là qui est intéressant. » (Manager 12)

"Quelque part ça me rassure aussi, si je n'ai rien, si tout va bien, alors que parfois, j'ai connu des époques où les systèmes d'informations n'étaient pas aussi performants, j'étais commercial itinérant, j'étais stressé parce que je n'avais pas accès à l'information. » (Directeur commercial 26)

... à tel point que la quasi-totalité d'entre eux n'envisagent plus de pouvoir travailler sans :

«Pour moi, c'est du bien-être. Ah non, non, non, moi je ne reviendrais pas en arrière. » (Commercial 19)

En parallèle du premier groupe d'exigences nouvellement engendrées par les TN, les personnes interrogées soulignent aussi l'aspect facilitateur de ces dernières, notamment en termes de réactivité et de fluidité des échanges, qui représentent alors des ressources nouvelles pour l'individu. Comme en témoignent les termes en gras des verbatims, ces ressources nouvelles sont vectrices de bien-être, d'efficacité au travail (et de réduction du stress) qui caractérisent le processus motivationnel modélisé par le JD-R (figure 3).

\section{Simultanéité du couple exigences-ressources}

Par ailleurs, cette dialectique inhérente aux effets des TN (plus d'exigences et de stress mais aussi plus de ressources, de bien-être et d'efficacité) se retrouve parfois directement évoquée par les personnes interrogées qui reconnaissent leur simultanéité :

«Ça a modifié considérablement la qualité de vie au travail. Disons que c'est différent, ce n'est plus comme avant. Il y a du mieux parce que l'on gagne du temps, c'est plus réactif, c'est peut-être plus rassurant. Après, c'est chronophage. Je pense qu'honnêtement on travaille plus, si on fait une étude, je ne sais pas mais comme ça, pour ma part je pense que je travaille plus avec les outils nomades que quand je n'en avais pas. " (Manager 21)

«J'essaye de me replonger douze mois en arrière. Je pense que de toute façon, ça entraine plus d'immédiateté dans les relations, donc forcément un stress supplémentaire évident. Après en termes de fonctionnement, de rapidité, il y a gain évident en termes d'organisation. Mais je pense qu'effectivement, ça génère un petit peu plus de stress au niveau des équipes. L'immédiateté dans le retour et dans la communication fait qu'on veut aller plus vite, trop vite. » (Manager 30) 
«Avant, quand il n'y avait pas mes mails sur le téléphone, je me connectais quand j'étais en déplacement, le matin, dès fois entre midi et deux et le soir. Alors que là, je suis connectée en permanence, je suis chez un client, je suis dans la salle d'attente, il a 5 min de retard, hop je regarde mes mails, je regarde ce qui est tombé, je fais suivre à un tel, un tel, donc on est dans l'immédiateté, pas dans l'immédiatement, mais en tout cas dans l'hyperréactivité au niveau de l'information avec ses avantages et ses inconvénients. »(Commercial 11)

«Par rapport à nos propres clients, ça nous donne une efficacité, une réactivité. Après le tort que l'on a parfois, du coup c'est d'aller trop vite, de survoler. C'est la face cachée des nouvelles technologies. » (Directeur commercial 22)

"Ça a un peu évolué, alors il y a des points négatifs et des points positifs. Le point positif, c'est qu'on est au courant plus vite des informations ou d'éventuelles relances clients ou de contacts clients qu'on doit faire. Par contre, le point négatif, c'est maintenant qu'on a cet outil, on attend de nous une réponse dans la minute. » (Commercial 24)

\subsection{Une invasion de la sphère privée par la sphère professionnelle couplée à plus d'autonomie et de flexibilité dans les deux sphères}

\section{Exigence - Disparition progressive de la frontière vie privée-vie professionnelle}

Avec l'arrivée des TN, la journée est de plus en plus rythmée par un enchevêtrement de séquences consacrées à la sphère privée et d'autres séquences à la sphère professionnelle. Cet état concerne également les espaces de travail avec une arrivée massive des tâches professionnelles dans les espaces privés, les TN participant ainsi à la disparition progressive de la frontière vie privée-vie professionnelle. La problématique de la multiplication des lieux de travail consubstantielle aux évolutions technologiques a été clairement identifiée et constitue un enjeu majeur pour l'ensemble des directions et des managers du territoire :

«Il faut savoir se respecter, et dans le respect, il y a aussi le respect des chartes d'utilisation de ces petits appareils (N.-B. : le smartphone). C'est vrai qu'il faut savoir parler boulot quand on parle boulot et quand c'est fini il faut savoir arrêter. Car on peut générer aussi de l'insatisfaction à être trop présent. Donc le bien-être au travail, ça passe aussi par le respect des périodes de non-travail.» (Manager 7)

«Après, pour déconnecter, je me rends inaccessible en allant dans des pays étrangers, avec tout le temps en tête "j'espère que, j'espère que, j'espère que ", et voilà c'est presque une source de stress et $j$ 'ai du mal à me dire même en vacances quand je suis en France, "je ne consulte pas mes mails aujourd'hui ». C'est compliqué. » (Directeur commercial 1)

Certaines des personnes interrogées regrettent ainsi le manque de moments de répit et souhaiteraient pouvoir faire une coupure nette après avoir quitté leur lieu de travail :

«Ce qui est prépondérant, ce serait de pouvoir être chez soi, sauf exception, à une heure raisonnable et d'être capable de pouvoir décrocher du bureau. C'est-à-dire, non seulement d'être là physiquement mais d'être là mentalement. » (Directeur commercial 22)

«Il n'y a plus de coupures, il n'y a plus d'heures, il y a un mélange des frontières entre la vie professionnelle et la vie privée, forcément. » (Commercial 2) 
«C'est le mauvais côté du nomadisme et des nouvelles technologies. C'est qu'après on est sans arrêt connecté dessus. ॥ (Commercial 33)

«J'ai un portable perso que j'ai depuis des années, que j'ai conservé, mais que j'ai limité au niveau du nombre d'heures, alors que celui qu'on a pour le boulot est en illimité, donc pendant mes vacances, $j$ 'allume celui du bureau de temps en temps pour téléphoner et je suis tentée de regarder mes mails et je suis tentée de répondre et de gérer les urgences. Donc en fait, je ne coupe jamais. » (Commercial 11)

Une nouvelle exigence des TN est donc la disparition des frontières spatiales et temporelles du travail. Cette disparition des frontières, cette hyperconnexion, est une source de stress et d'insatisfaction pour les personnes interrogées qui, pourtant, arrivent également à en extraire de nouvelles ressources.

\section{Ressource - Autonomie et flexibilité au travail}

Les entretiens menés au sein des équipes commerciales de La Poste font également apparaitre l'importance des thématiques relatives à l'organisation spatio-temporelle du travail lorsque l'on aborde la question du BET. L'autonomie est l'une des caractéristiques dominantes des différents profils de postes constituant les équipes commerciales de La Poste, à laquelle l'ensemble des interviewés est profondément attaché. Or, le déploiement des TN a renforcé cette dernière au détriment d'un contrôle plus direct, mais a en contrepartie également modifié le rapport au temps des salariés. Ainsi, la notion de temps de travail en termes de durée et de stabilité des horaires est exprimée à l'aune de ces modifications. Les débordements en termes d'organisation spatio-temporelle que peuvent occasionner les TN sont relativement bien acceptés car en partie compensés par les libertés d'action dont disposent les membres des équipes commerciales. Cette idée se retrouve aussi bien dans la population des managers...

«Après, c'est super pratique, parce que moi si j'ai un imprévu, automatiquement, je peux y aller et puis m'occuper et le faire un peu plus tard. Ça laisse de la souplesse. » (Manager 21)

... que dans celle des commerciaux :

«À certains moments, effectivement, la qualité de vie au travail est mise à mal par certaines façons d'organiser le travail. Oui mais bon, c'est le jeu. On est cadre et on l'accepte parce que justement vendredi, il y a le carnaval et du coup je peux m'en aller plus tôt, ou alors j'ai mal aux dents et le dentiste ne peut me prendre qu'à 16 h et ben j'y vais ce jour-là et après je bosse chez moi, ou alors le lendemain je vais venir plus tôt et partir plus tard.» (Commercial 4)

\section{Ressource - Flexibilité dans l'articulation vie privée-vie professionnelle}

Plusieurs personnes interrogées considèrent que les TN viennent favoriser l'articulation vie privée-vie professionnelle. En conséquence, le modèle des générations précédentes basé sur une séparation nette des différentes sphères de vie n'est ni envié, ni regretté, comme en attestent les dires de cette manager des ventes âgée de 45 ans (manager 12) :

«Il n'y a plus de frontières ou alors elle est en zigzag, mais ça ne me perturbe pas moi. Je vois bien que je ne sors pas du travail et que j'ai fini, comme la référence que j'avais de mes parents qui effectivement quand ils étaient hors boulot, ils n'étaient plus au boulot, ils n'avaient pas d'appel du bureau, ils n'avaient rien. Aujourd'hui, mes enfants me voient avec des appels du bureau, parler boulot, quand je peux être à la maison, un jour de RTT ou un jour où je ne travaille pas. 
Mais moi, pour moi, ça ne me choque pas, parce qu'une fois que j'ai raccroché je suis de nouveau là, ça ne me prend pas des lustres. »

De même, les interviewés témoignent de l'effet facilitateur des TN, en termes de conciliations des temps de vie (par exemple la gestion de l'état de santé ou des activités des enfants, l'achèvement de dossiers professionnels incompatible avec les obligations parentales) et apprécient de pouvoir travailler au domicile pour bénéficier du calme et être plus efficaces dans leur travail :

«Moi, je suis beaucoup plus opérationnelle, performante, assidue, quand je travaille de chez moi, c'est-à-dire presque je ne vais pas me lever de ma chaise pour aller aux toilettes, alors que quand je suis au bureau, j'aurais tendance à me disperser. » (Commercial 19)

"Mais non ça n'empiète pas du tout, après ça t'offre une telle liberté que... moi hier soir, j'avais les courses à faire, j'ai bossé jusqu'à $21 \mathrm{~h}$, ça ne me dérange pas du tout, et ça n'a dérangé personne. Pour moi, ça n'empiète pas dans ma vie privée. » (Commercial 19)

"Personne ne m'impose de regarder mes mails à 22 h 30 ou à n'importe quelle heure. Je le fais parce que je le veux bien donc je ne le ressens pas comme un stress ou un poids sur mon activité. Après, par contre, ce qui est intéressant, c'est le fait de pouvoir travailler de chez soi, pour tout ce qui est création de chiffrage, etc., ça par contre c'est un vrai, vrai plus, un très, très gros avantage. C'est vraiment très pratique. » (Commercial 33)

Cette nouvelle exigence de la porosité des frontières entre vie professionnelle et vie personnelle est à la fois source de stress et d'insatisfaction liée à la surconnexion, mais aussi de liberté et de performance pour les individus qui la vivent. Elle engendre conjointement de nouvelles ressources venant réduire son coût psychologique et organisationnel, en donnant de l'autonomie et de la flexibilité au travail, mais aussi dans la vie quotidienne. Les deux processus identifiés dans le modèle JD-R sont ainsi illustrés par ce deuxième couple d'exigences-ressources pouvant de plus exister conjointement chez les personnes interrogées.

\section{Simultanéité du couple exigences-ressources}

Comme pour le premier groupe d'exigences-ressources identifiées, les personnes interrogées ont parfois conscience du caractère ambivalent de cette porosité des frontières spatiales et temporelles du travail et de la vie personnelle. Ils ressentent alors simultanément les effets des TN en termes de bien-être et de stress qui peuvent alors coexister de façon presque indépendante.

«On peut être plus réactif, répondre plus facilement, par contre, le bémol que je mets c'est sur ce que j'évoquais au début, il faut être vigilant à ne pas se faire complètement phagocyter parce qu'alors là, pour le coup, on a l'impression de ne jamais décrocher. » (Commercial 23)

«Il y a le pour et le contre. Je trouve que pour certains aspects, ça facilite la conciliation vie professionnelle-vie personnelle. Par exemple, un jour comme aujourd'hui, je ne suis pas obligée de repasser au bureau. Donc ça quelque part c'est une bonne conciliation. Après le pendant, le risque, c'est que l'on a nos mails en permanence, week-end compris, soirées comprises, tout le temps, et que du coup si on ne met pas une certaine limite, on pourrait ne plus s'arrêter. » (Directeur commercial 22) 
"C'est le cercle vicieux ou vertueux, c'est comme on veut, c'est-à-dire plein d'avantages d'un côté, parce que le télétravail sans les outils nomades de fait n'existe pas, alors qu'il permet un degré d'autonomie en termes d'organisation qui est quand même sympa. Après, on a toujours du mal à définir si le fait que l'on n'arrive pas forcément à s'arrêter est dî̀ à soi ou à ce qu'inconsciemment cela induit. » (Manager 28)

Les différents échanges avec les personnels du groupe La Poste (en entretien et lors des observations) révèlent enfin un dernier couple exigences-ressources important que nous développons dans la dernière section.

\subsection{Un effort de synthèse de masses d'informations facilité par leur accessibilité}

\section{Exigence - Surcharge d'informations à traiter et synthétiser}

D'un point de vue qualitatif, l'augmentation de la charge de travail liée aux TN se manifeste dans le fait de devoir développer ou approfondir certaines compétences pour optimiser l'utilisation des outils mis à disposition (solutions matérielles ou logicielles) mais aussi pour traiter de manière efficiente l'ensemble des informations transitant. Par exemple, devant le phénomène "d'infobésité " auquel les équipes sont confrontées, certains managers ont dû développer un fort esprit de synthèse et pour s'assurer que les informations principales sont bien assimilées, proposent des résumés de documents :

«Moi, j'estime qu'en tant que manager, justement pour leur amener des choses, parce que je sais que certains ne lisent pas tout, je décante un peu pour eux. D'ailleurs, je titre mon mail : "j'ai lu pour vous ». » (Manager 12)

«Concrètement, il y a un côté profusion d'informations, trop plein d'informations, nécessité d'une réactivité. Ça a quand même beaucoup changé, le temps que l'on nous donne pour réagir à une information, pour la trier, pour l'organiser, pour la gérer est super court. » (Directeur commercial 22)

«Des mails, on en reçoit souvent beaucoup et souvent trop, et moi ça me prend un peu de temps. Mais sur la multitude de mails que je reçois, avant de transmettre à l'équipe, je prends le temps de lire le mail, et je le transmets que s'il y a un réel intérêt. S'il y en a pour dix minutes de lecture et qu'il n'y a qu'une phrase qui a vraiment un intérêt pour eux, je la mets en exergue je leur dis "voilà ce qui est intéressant à retenir » qu'ils ne perdent pas tous de temps à lire, ou pas le lire carrément. [...] J'essaie de supprimer les mails style "pour info » avec trois points de suspension, ça c'est vraiment le travers du mail. Dès fois, les informations importantes passent complètement au travers, elles sont complètement dissolues dans tout le reste, et si on voulait vraiment tout lire comme il faut et tout approfondir, on passerait deux à trois heures par jour, et ce n'est pas possible. Ça va à l'encontre de la réactivité. Moi ça me prend un peu de temps mais eux ils me disent que ça leur en fait gagner beaucoup. » (Manager 9)

Cette exigence de synthèse concerne principalement les managers dans la mesure où les opérationnels n'ont pas le temps de gérer et digérer le surplus d'informations disponibles par les TN. Cette exigence est toutefois compensée par un accès rapide aux informations pertinentes. 


\section{Ressource - Accessibilité des informations}

La facilité d'accès à une grande quantité de données, et la mise à disposition d'un grand nombre de ressources sont également mises en avant et constituent un atout indéniable dans la réalisation des tâches quotidiennes (échange, stockage, accès aux données, reporting...).

« Je trouve qu'effectivement, quand je regarde l'accès à des données relativement facilement, je me sens beaucoup plus efficace dans mon travail et donc comme je me sens beaucoup plus efficace on peut dire que ça a amené du confort. » (Directeur commercial 13)

«Ça a apporté effectivement un plus au niveau de la qualité de vie au travail, c'est quand même un confort, soit de regarder son agenda, soit de consulter ses mails, c'est quand même un confort dans notre travail, ça nous supprime un petit peu de stress. [...] On voit un mail qui est assez urgent donc on peut le traiter directement sur le smartphone, on n'a pas le stress de dire " je n'aurai pas le temps de le faire». Ça m'apporte une réactivité vis-à-vis de mon travail. » (Commercial 26)

«Ça a influencé beaucoup de choses, l'accès directement à l'information qui fait que l'on n'a plus besoin de la retenir, la communication directe, le lien avec la famille, les enfants. On sait que l'on peut être consulté très facilement. [...] On utilise l'email quand ce n'est pas important, le SMS quand c'est important, le téléphone quand c'est complexe. On a plusieurs canaux de communication, et on les utilise en fonction du niveau d'urgence. Et ça, c'est quand même un plus, un gros plus. » (Commercial 2)

Cette facilité d'accès à l'information semble donc contribuer au processus motivationnel de l'introduction des TN (figure 3) en améliorant le confort de travail et l'efficacité tout en contribuant à réduire le stress du métier.

\section{Simultanéité du couple exigences-ressources et risque d'une hyperconnexion addictive}

Certains commerciaux relèvent même parfois la simultanéité des deux processus : une difficulté à gérer le surplus d'informations compensée par la réactivité accrue d'un accès facile à une grande quantité d'informations :

«Cela permet d'être bien plus réactif, plus au courant des choses. Après, effectivement, il y a le contrecoup, on a tendance à être noyé sous les informations, à avoir plein d'informations de partout et ça, on a du mal à les emmagasiner puis à s'en souvenir. Mais sinon je dirais que cela va plutôt dans le bon sens, en tout cas, pour nous en tant que commercial, c'est plutôt des avantages. » (Commercial 33)

On peut donc avoir une situation d'augmentation conjointe du stress et du bien-être au travail par l'usage des TN, situation pour laquelle un équilibre n'est pas facile à trouver chez les individus, oscillant parfois entre une hyperconnexion facilitatrice et une hyperconnexion addictive.

Le résultat des entretiens révèle que le traitement d'une forte masse d'informations via les TN peut conduire à l'addiction chez certaines personnes. Le rôle facilitateur des $\mathrm{TN}$, et notamment du mobile multifonction quant aux traitements des informations par messageries instantanées, entraine des changements comportementaux qui deviennent parfois excessifs. Ainsi, certains utilisateurs font de la consultation durant la nuit... 
«Moi, je le fais à deux heures du matin, trois heures du matin. C'est vrai que c'est facilitateur. » (Directeur commercial 21)

... d'autres relèvent leurs mails au moins deux fois par heure jusque tard le soir, ou tentent de le faire à l'insu de leur entourage :

«Je n'arrive pas à me déconnecter du truc... mais disons, qu'en gros, tant que je ne fais pas choper par ma femme, ce n'est pas grave. Et oui, parce que je les regarde, je les transfère, ça va hyper vite. »(Commercial 8)

Certains utilisateurs évoquent eux-mêmes un phénomène d'addiction :

«On sait très bien que ce n'est pas indispensable, on sait très bien que l'on peut ne pas l'allumer, on sait très bien que l'on peut ne pas le regarder. C'est une question d'envie, c'est pour cela que l'on parle d'addiction, c'est parce que c'est plus fort que nous. Rien ne nous y oblige mais c'est un réflexe. C'est bien plus profond qu'une question d'envie, c'est un réflexe. » (Commercial 2)

Ce risque d'addiction matérialise à l'extrême une situation dans laquelle les deux processus identifiés par le JD-R existent simultanément pour un même individu. Pour conclure cette section, le tableau 3 synthétise l'ensemble de nos résultats. Dans la section suivante, nous discutons ces derniers au regard de la littérature et du modèle JD-R présenté en figure 3 et formulons plusieurs propositions de recherche sur les relations entre bien-être et stress technologique au travail. 
Tableau 3. Synthèse des résultats présentant la simultanéité des couples exigences-ressources identifiés

\begin{tabular}{|c|c|c|c|c|}
\hline Exigences & Réactivité accrue par des échanges plus fluides & \multirow[t]{3}{*}{ Autonomie et flexibilité au travail } & \multirow[t]{3}{*}{$\begin{array}{l}\text { Flexibilité dans l'articulation vie } \\
\text { privée-vie professionnelle }\end{array}$} & \multirow[t]{4}{*}{ Accessibilité des informations } \\
\hline $\begin{array}{l}\text { Augmentation de la } \\
\text { charge de travail }\end{array}$ & $\begin{array}{l}\text { Profusion d'informations, accumulation de } \\
\text { sollicitations, interruptions d'activités } \\
\rightarrow \text { Travail supplémentaire } \\
\text { Intensification des échanges et pression de la } \\
\text { réponse } \\
\rightarrow \text { Exigence de réactivité }\end{array}$ & & & \\
\hline $\begin{array}{l}\text { Immédiateté et pression } \\
\text { de la réponse }\end{array}$ & $\begin{array}{l}\text { fluidification des échanges, rassurant pour les } \\
\text { individus } \\
\rightarrow \text { Les TN comme ressources permettant } \\
\text { d'être plus réactif et « rassuré » }\end{array}$ & & & \\
\hline $\begin{array}{l}\text { Disparition progressive } \\
\text { de la frontière vie privée- } \\
\text { vie professionnelle }\end{array}$ & & \multicolumn{2}{|c|}{$\begin{array}{l}\text { Arrivée massive des tâches professionnelles dans les espaces privés, manque } \\
\text { de moments de répit, pas de coupures nettes } \\
\rightarrow \text { Disparition des frontières spatiales et temporelles du travail source de } \\
\text { stress } \\
\text { Augmentation des libertés d'action et d'organisation du travail et de sa vie } \\
\text { personnelle } \\
\rightarrow \text { Les TN comme ressources pour mieux organiser son travail et sa vie } \\
\text { personnelle (travail asynchrone, autonomie, conciliation des temps de } \\
\text { vie) }\end{array}$} & \\
\hline $\begin{array}{c}\text { Surcharge } \\
\text { d'informations à traiter } \\
\text { et synthétiser }\end{array}$ & & & & $\begin{array}{l}\text { Exigence d'esprit de synthèse } \\
\text { pour digérer le volume } \\
\text { supplémentaire d'informations } \\
\text { à traiter (surtout pour les } \\
\text { managers) } \\
\text { Accessibilité des informations } \\
\text { qui rend le travail plus efficace } \\
\text { (ressource) } \\
\text { Phénomène d'addiction }\end{array}$ \\
\hline
\end{tabular}




\section{Discussion : adaptation du modèle JD-R aux effets des TN}

Les résultats de cette étude qualitative suggèrent que l'introduction de $\mathrm{TN}$ engendre conjointement du stress et du bien-être au travail. Sur ces bases, nous détaillons d'abord plusieurs propositions de recherche découlant de la confrontation de nos résultats avec ceux de la littérature (5.1 à 5.3). Nous justifions et proposons ensuite une adaptation du modèle JD-R révisé à l'introduction des technologies nomades, nous permettant d'avancer et de discuter plusieurs pistes de recherches futures (5.4).

\subsection{Une réactivité accrue liée aux caractéristiques technologiques pour plus de BET}

La charge de travail supplémentaire induite par l'utilisation des TN (Bretesché, Corbière et Geffroy, 2012 ; Chevalet et Moatty, 2012 ; Greenan et al., 2012 ; Gupta, Li et Sharda, 2013 ; Klein et Long, 2013 ; Venin, 2013) ainsi que les pressions d'une réponse immédiate apparaissent comme des exigences professionnelles nouvelles. Ces exigences, comme le soulignent les personnes interrogées de façon assez transparente, sont souvent génératrices de stress (Ayyagari, 2007 ; Hung, Chang et Lin, 2011 ; Peyrat-Guillard et Samier, 2004 ; Poole et Denny, 2001). Mais parallèlement, les personnes interrogées mentionnent une amélioration du bien-être au travail en raison de la réactivité permise par les TN.

Nos résultats mettent en avant que certaines caractéristiques des technologies peuvent avoir un effet positif sur le BET de l'individu. L'importance des caractéristiques technologiques trouve un large écho dans la littérature, notamment au travers du modèle TAM (modèle d'acceptation de la technologie) proposé par Davis, Bagozzi et Warshaw (1989), au sein duquel les variables « utilité perçue » et « facilité d'utilisation perçue » jouent un rôle essentiel pour expliquer l'utilisation d'un système technologique. Ces variables sont importantes, car elles influencent l'individu sur la perception de son efficacité personnelle et l'amélioration de sa performance. Un parallèle peut ainsi être fait avec la vision eudémonique du bien-être, et la notion de réalisation de soi (Ryan et Deci, 2001).

$\mathrm{Au}$ sein des équipes commerciales de La Poste, l'arrivée du mobile multifonction et des PC portables-tablettes était attendue par une grande majorité des individus, notamment en raison des caractéristiques de ces outils (facilité d'utilisation perçue, utilité perçue et fiabilité) et également parce que ces outils technologiques paraissent être en adéquation avec de nombreuses activités effectuées régulièrement. Ces caractéristiques technologiques ont permis aux individus d'améliorer leur réactivité, particulièrement pour ne pas rater une affaire commerciale (il est apparu rassurant de se savoir joignable à tout moment et de pouvoir agir également rapidement sans problème technique sur les TN). Par conséquent, dans notre contexte de recherche, il semble exister un bon alignement entre les fonctionnalités offertes par les TN déployées et les activités des utilisateurs, ce qui rejoint la notion de fit proposé par Goodhue et Thompson (1995), défini comme étant "le degré avec lequel une technologie assiste un individu dans la réalisation de son portefeuille de tâches ». Or, comme le précisent Ayyagari, Grover et Purvis (2011), si les individus estiment des technologies utiles, ces dernières améliorent leur capacité à effectuer des tâches plus rapidement ou à être plus productifs, favorisant ainsi le BET.

Proposition 1. Une amélioration du BET via une meilleure réactivité liée notamment aux caractéristiques technologiques des TN compense l'augmentation de stress due à la surcharge de travail et à la pression de la réponse. 


\subsection{Autonomie et flexibilité, sources de BET}

À l'inverse, certaines ressources identifiées (autonomie et flexibilité) semblent avoir un double rôle de génération de bien-être technologique d'une part (Korunka, Zauchner et Weiss, 1997 ; Travaglianti, 2011), et de réduction du stress technologique d'autre part (Ragu-Nathan et al., 2008 ; Venin, 2013), tandis que la porosité entre vie privée et vie professionnelle engendrée par les TN favorise le stress et les tensions chez les individus.

Sur ces aspects, nos résultats rejoignent ceux de Cucchi (2014) qui évoque la mise en tension professionnelle permanente induite par les $\mathrm{TN}$ du fait que leur utilisation au domicile favorise le conflit travail-famille et suscite des conflits de rôles. L'auteur insiste également sur la dualité provoquée par les TN sur le phénomène du conflit travail-famille, famille au sein de laquelle l'individu est présent physiquement, mais fait preuve d'une absence émotionnelle et sociale. Certains verbatims reflètent ce constat ("Mon conjoint m'a dit : " ou tu t'occupes de ton boulot, ou tu es à la maison" (commercial 16); "Moi, j'ai une ado qui m'a dit : " mais maman, tu fais toujours des devoirs à la maison » (commerciale 23) ; "Quand je suis en train de lire mes mails à la maison, je n'écoute pas trop ce qui est en train de se passer autour 》 (directeur commercial 31). Cette sensation d'intrusion dans la vie privée est également évoquée par Besseyre des Horts et Isaac (2006) au travers de témoignages de salariés utilisateurs de TN professionnelles. Bérard (2015) insiste sur le rôle des technologies concernant l'élargissement du temps de travail qui colonise toujours plus le temps libre. Ayyagari, Grover et Purvis (2011) mettent en avant un effet significatif des TN sur le conflit travail-famille, mais constatent une tolérance des individus par rapport à l'invasion des TN dans la sphère privée, notamment en raison des normes existantes ou des attentes en termes de productivité.

Malgré ce sentiment de débordement que peuvent parfois ressentir les membres des équipes commerciales, notre analyse qualitative a également montré que les TN restaient fortement plébiscitées pour les facilités organisationnelles et l'autonomie qu'elles offrent. L'existence de ces nouvelles ressources (flexibilité et autonomie) se retrouvent également dans la littérature, comme lorsque Benedetto-Meyer et Klein (2012) interrogent la capacité des TN à améliorer la conciliation travail-famille. Derks, Zecic et Bakker (2010), dans un travail portant sur l'influence des mobiles multifonctions sur le bien-être des salariés, montrent que ces outils technologiques favoriseraient la productivité et la flexibilité des salariés, permettant aux travailleurs une meilleure gestion de l'équilibre travail-famille. Plus récemment, Derks et al. (2016) ont conduit une étude quantitative, dont l'objectif est la mise en lumière de la relation entre l'utilisation du téléphone professionnel en dehors des horaires de travail et le conflit travail-famille. Les auteurs montrent que l'usage du téléphone en dehors des horaires de travail peut aider l'individu à répondre simultanément aux exigences de son travail et à celles de sa famille, pouvant ainsi réduire le conflit travail-famille.

Dans cette logique, nos résultats mettent en exergue le caractère à la fois contraignant et facilitateur des TN en montrant que leur introduction dans l'organisation peut être à double tranchant. Ce point renvoie aux travaux de plusieurs auteurs (Day, Scott et Bakker, 2010 ; Lowry et Moskos, 2005 ; Towers et al., 2006) qui expriment conjointement les effets positifs de la flexibilité et de la perméabilité liées à l'utilisation de technologies mobiles sur l'équilibre travail-famille, mais également la possibilité d'une augmentation de la disponibilité du salarié pour le travail, entrainant un empiètement sur le temps consacré à la famille.

Dans nos résultats, nous avons identifié que les TN semblent permettre de gérer l'arbitrage autonomie-contrôle à travers la disponibilité et l'accessibilité quasi-permanente des individus. Ces effets sont évoqués par Gollac, Greenan et Hamon-Cholet (2000) qui soulignent que les salariés informatisés voient autonomie et contrôle se renforcer conjointement. Gheorghiu et Moatty (2005), pour leur part, parlent d'autonomie encadrée. Ainsi, lors du déploiement de 
technologies mobiles, il semble important pour les organisations de s'assurer d'un maintien des marges de manœuvre suffisant pour les salariés, afin que l'autonomie reste une ressource organisationnelle mobilisable en cas de menace sur le BET. Ces éléments nous conduisent à avancer la proposition suivante :

Proposition 2. Le stress engendré par la disparition des frontières entre vie privée et vie professionnelle à la suite de l'introduction de TN est compensé quand ces dernières donnent également plus d'autonomie et de flexibilité aux individus, sources de BET.

\subsection{Une meilleure accessibilité et de nouvelles compétences génératrices de BET... et d'addiction}

La surcharge à laquelle est soumise notre échantillon d'étude est principalement de nature informationnelle et communicationnelle. Pour Helmersen et al. (2001), la surcharge informationnelle fait référence aux difficultés liées à la recherche, au stockage, et au traitement d'un volume important d'informations disponibles. Elle constitue avant tout une source de stress pour l'individu qui la subit, en raison notamment de la quantité toujours plus importante d'informations à gérer (Autissier et Lahlou, 1999), dans des délais qui se raccourcissent constamment (Denis et Assadi, 2005 ; Farhoomand et Drury, 2002 ; Metzger et Cléach, 2004). La surcharge communicationnelle, quant à elle, se manifeste par un fort accroissement des échanges entre différents collègues de travail, notamment via les emails, générant une sursollicitation (Denis et Assadi, 2005 ; Helmersen et al., 2001 ; Saintive, 2000).

Ainsi, les TN sont susceptibles d'engendrer une perte de sens pour le salarié qui peut voir son activité cognitive amputée (Gomez et Chevallet, 2011), mais elles peuvent également faciliter l'accomplissement de certaines tâches et contribuer ainsi à un gain de temps, mis à profit pour l'accomplissement d'activités créatrices de valeur (GRePS-Apec, 2011). Dans cette perspective, notre étude a notamment permis de constater que des nouvelles compétences ont émergées par l'utilisation des TN, comme par exemple : une meilleure gestion du planning, la gestion des relations à distance et un travail de synthèse de la part de certains managers à destination de leur équipe relativement aux informations circulant par mails collectifs. L'arrivée des TN semble donc avoir favorisé l'évolution des compétences des membres des équipes commerciales de La Poste. Ces résultats complètent ceux de Joling et Kraan (2008) qui montrent que les utilisateurs d'ordinateur ont plus fréquemment des possibilités d'apprentissage et ceux de Greenan et al. (2012) qui mettent en avant que l'utilisation des TN accompagne l'acquisition et la mobilisation de nouvelles compétences. À la suite de nos résultats et du retour à la littérature ci-dessus, nous avançons que le stress lié à l'exigence de surcharge informationnelle peut être contrebalancé par une augmentation du BET liée à de nouvelles ressources apportées par les TN (une meilleure accessibilité des informations et un développement de nouvelles compétences). Il en découle la proposition suivante :

Proposition 3. Le stress généré par la surcharge informationnelle et communicationnelle liée aux TN est compensé par une accessibilité accrue aux informations pertinentes et par le développement de nouvelles compétences génératrices de BET.

Nos résultats illustrent l'importance d'accompagner l'introduction de TN d'une réflexion sérieuse quant à la disponibilité des « bonnes » informations (impliquant un travail de synthèse et de choix en amont) et à la montée en compétences des individus.

Enfin, un des éléments paroxystiques de cette proposition est le développement d'un phénomène d'addiction en lien avec la surcharge informationnelle et communicationnelle à 
l'instar de la dépendance aux réseaux sociaux (Kefi, Mlaiki et Kalika, 2016). Notre analyse qualitative a en effet mis en évidence des comportements excessifs en termes d'utilisation du téléphone professionnel par un certain nombre de personnes interviewées. Ces dernières ont évoqué spontanément le terme d'addiction, pour caractériser la relation qu'elles entretiennent avec leur mobile multifonction, notamment pour ce qui est de la consultation de la messagerie professionnelle. Cela vient confirmer la place centrale tenue par les outils de communication, dont le téléphone est le principal support, qui deviennent une pierre angulaire autour de laquelle s'organise la journée de travail. Ces divers constats rejoignent ici différents travaux de recherche sur le sujet (Ayyagari, Grover et Purvis, 2011 ; Cucchi, 2014 ; de la Rupelle, Fray et Kalika, 2014 ; GRePS-Apec, 2011 ; Vaghefi et Lapointe, 2013).

Alors que Oulasvirta et al. (2012) ont étudié des sujets qui vérifient inutilement leur téléphone, juste par habitude (jusqu'à 34 fois par jour), ce qui peut conduire à une addiction au téléphone intelligent, Lee et al. (2014) observent que pour les utilisateurs ayant un comportement excessif dans l'usage du téléphone, celui-ci est souvent la première chose qu'ils regardent au lever et la dernière avant de se coucher. De manière analogue, dans notre étude, certaines habitudes de consultations excessives ont été révélées : consultation de la messagerie mobile toutes les demiheures jusqu'à 23 heures, relevé des mails durant la nuit, consultation de la messagerie durant des activités sportives ou de loisir... Si l'on s'en réfère à la grille de lecture offerte par Beaudry et Pinsonneault (2005), le phénomène d'addiction pourrait éventuellement être perçu comme un usage déviant, au travers duquel l'individu, après une analyse de la situation en termes de bénéfices et menaces, s'appuie sur la ressource que constitue le téléphone professionnel, et adopte une stratégie qu'il considère adaptée pour répondre à ses objectifs professionnels. En résulte la coexistence de stress et de bien-être technologiques à des niveaux significatifs, indépendamment du fait que les ressources permettent de réduire la pression des exigences et donc le niveau de stress. En complément des premiers constats de Korunka, Zauchner et Weiss (1997) et Ragu-Nathan et al. (2008), nos résultats évoquent ainsi un découplage entre stress et bien-être technologiques qui ne sont donc pas les deux faces d'une même médaille mais bien des manifestations simultanées de l'influence des TN sur les individus au travail.

En résumé, le temps de travail se contractant (le travail est plus dense et plus intense avec les TN), les exigences portant sur les salariés s'accroissent donc tandis que les TN déchargent les individus d'un certain nombre de limites spatiales et temporelles, tant dans leur vie professionnelle que leur vie privée. Elles facilitent l'acquisition des connaissances, l'accès rapide à l'information utile et permettent ainsi aux individus de gagner en efficacité professionnelle et personnelle par une plus grande autonomie et flexibilité. Les TN engendrent donc des ressources mobilisables par les individus pour réduire la pression du travail quotidien et prendre des décisions plus rapides. Les $\mathrm{TN}$ ont un double effet : elles contraignent l'individu par l'augmentation de l'intensité du travail mais lui permettent en même temps d'améliorer son efficacité et de réduire la pression relative à la recherche et au traitement de l'information. In fine, elles sont donc perçues comme engendrant du stress mais aussi comme améliorant le bien-être au travail et la performance individuelle et organisationnelle.

\subsection{Un modèle JD-R adapté à l'introduction des $\mathrm{TN}$ : vers de nouvelles pistes de recherche}

Nos résultats permettent une adaptation du modèle JD-R révisé à l'introduction des technologies nomades. Les verbatims nous ont permis d'identifier des couples d'exigences- 
ressources pouvant augmenter simultanément le stress et le bien-être au travail des individus, rompant avec l'idée que stress et bien-être évoluent systématiquement en sens inverse comme le suggère le modèle JD-R révisé (Schaufeli et Taris, 2014) présenté en section 2.4. Comme le recommandent Schaufeli et Taris (2014, p. 57) dans leur bilan et perspectives de l'utilisation du modèle JD-R dans les recherches académiques, «le processus de dégradation de la santé et le processus motivationnel doivent être étudiés conjointement $»$. Notre étude fait en effet ressortir l'importance de ces deux processus après l'introduction de TN au sein du groupe La Poste. La figure 4 présente le modèle JD-R qui émerge à l'issue de notre analyse des résultats.

Nous avons reporté dans cette figure les exigences et ressources apportées par les TN, en les regroupant en trois couples exigences-ressources cohérents, c'est à dire ayant une origine commune : 1) augmentation de la charge de travail et pression de la réponse versus réactivité accrue, 2) disparition des frontières entre vie privée et vie professionnelle versus autonomie et flexibilité professionnelle et personnelle, et 3) surcharge d'informations à traiter versus accessibilité des informations. Comme dans le modèle JD-R révisé (Schaufeli et Bakker, 2004 ; Schaufeli et Taris, 2014), les exigences sont génératrices de stress (relation R1 sur le graphique) tandis que les ressources permettent d'augmenter le bien-être au travail (relation R2). De même, nos résultats sont en adéquation avec ceux bien établis de la littérature concernant les relations R3 (entre ressources et technostress) et R4 (entre BET et performance au travail). Toutefois, dans le cas des TN, les couples d'exigences-ressources ont un effet simultané (relation R5) qui découple les conséquences des TN sur le stress et le bien-être technologique. Les deux états (et leur intensité) n'évoluent pas nécessairement en sens inverse puisqu'une situation de stress technologique n'est pas incompatible avec une situation de bien-être technologique (relation R6). Il s'agit ici d'un résultat important de cette étude qualitative. 
Figure 4. Modèle JD-R issu des résultats

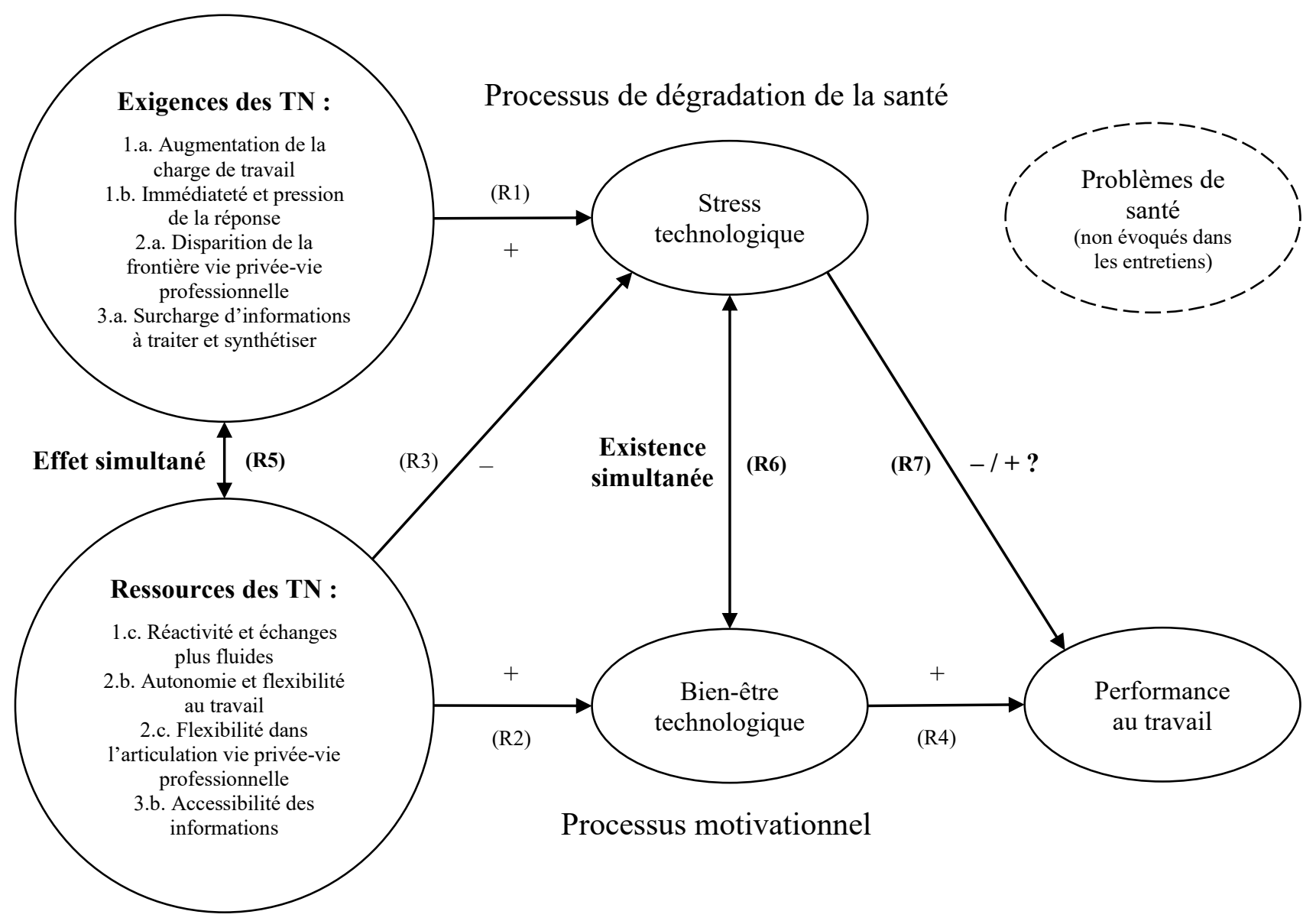

Une autre question émerge des résultats sur le sens et l'intensité de la relation entre stress technologique et performance au travail (relation R7). Le sens de cette relation est originellement inverse mais nos résultats ne permettent pas d'exclure une relation positive entre les deux concepts, selon le type d'exigence qui engendre l'augmentation du stress. En effet, la littérature sur le JD-R a progressivement mis en évidence deux catégories d'exigences (Crawford, LePine et Rich, 2010 ; Van den Broeck et al., 2010) qui agissent en sens inverse sur l'engagement au travail et donc la performance. Les auteurs distinguent les exigences « challenges » des exigences « obstacles ». Les exigences « challenges » sont susceptibles de favoriser le développement personnel, la maitrise de son travail et la performance (engendrant $\mathrm{du}$ stress positif) tandis que les exigences « obstacles » sont réellement bloquantes pour le développement de l'individu et l'atteinte de ses objectifs (engendrant un stress négatif venant réduire la performance au travail). Schaufeli et Taris (2014) proposent même une redéfinition des notions d'exigence et de ressource au regard de ces deux catégories en classant les exigences « challenges » en ressources.

En conséquence et perspective de recherche, le sens et l'intensité de la relation entre stress technologique et performance au travail n'est pas théoriquement déterminé et mérite une étude empirique supplémentaire. Il s'agirait de déterminer la nature des exigences mentionnées par les personnes interrogées dans cette étude qualitative. Les conséquences du stress technologique en termes de performance au travail pourraient ainsi être estimées et expliquées au regard de ce JD-R révisé et adapté aux TN en fonction de la nature des exigences qu'elles engendrent. 


\section{Conclusion}

Cet article présente les résultats d'une étude qualitative s'intéressant à l'influence de l'introduction de technologies nomades sur le stress, le bien-être et la performance au travail. Il s'agit d'une étude qualitative reposant sur des entretiens semi-directifs et de l'observation flottante auprès de managers, commerciaux et directeurs commerciaux du groupe La Poste à la suite de l'introduction de TN dans une grande zone géographique du sud de la France.

Plusieurs résultats importants émergent de cette étude. Dans un premier temps, il ressort de façon assez intéressante et nouvelle que l'introduction de technologies nomades engendrent conjointement une augmentation du bien-être et du stress technologique. Cette situation de découplage entre stress et bien-être s'explique par le fait que les TN transforment les frontières, le contenu et l'intensité du travail de façon profonde. En effet, dans un deuxième temps, les entretiens ont permis d'identifier plusieurs couples d'exigences-ressources issues des transformations des situations de travail induites par les technologiques nomades. Ces couples d'exigences-ressources, dans la perspective théorique du modèle JD-R (Demerouti et al., 2001 ; Schaufeli et Bakker, 2004), traduisent le fait que les TN deviennent des facilitateurs du travail, sources de bien-être, en même temps qu'elles rendent le travail plus exigeant et intense, source de stress. Ainsi, en même temps qu'elles augmentent la charge de travail, l'immédiateté des échanges et la pression de la réponse, elles permettent aux individus d'être plus réactifs et d'échanger de l'information de façon beaucoup plus fluide. De plus, si les TN entrainent une disparition des frontières entre vie professionnelle et vie privée, elles donnent aux individus une plus grande autonomie et une plus grande flexibilité professionnelle et personnelle. Enfin, les TN sont responsables du phénomène d' "infobésité » nécessitant un effort de synthèse et de traitement d'une quantité importante d'informations, tout en facilitant largement l'accès aux informations pertinentes liées aux activités professionnelles. Dans un dernier temps, nos résultats permettent d'aboutir à un modèle théorique JD-R adapté à l'introduction des TN.

Par ces résultats, notre étude contribue de plusieurs façons à la littérature étudiant l'influence des TN sur les individus en situation de travail. Premièrement, l'étude qualitative a permis l'identification claire de couples exigences-ressources liés à l'introduction des TN et sources à la fois de stress technologique mais aussi de bien-être associé à une meilleure performance. Deuxièmement, à travers ces couples exigences-ressources, les résultats mettent en évidence la présence de stress et de bien-être au travail de façon simultanée avec, à l'extrême, des comportements d'addiction. Il existerait donc un découplage entre le stress et le bien-être technologique lors de l'introduction de $\mathrm{TN}$, les phénomènes d'addiction représentant une situation extrême de bien-être et de stress intense associés à l'utilisation des TN. Troisièmement, nous contribuons à la littérature mobilisant le modèle JD-R révisé (Schaufeli et Taris, 2014) en développant un modèle JD-R adapté à l'introduction des TN. Ce modèle invite les recherches futures à tenir compte de la proposition de Schaufeli et Taris (2014) de redéfinir le statut des exigences selon qu'elles sont des « challenges» ou des « obstacles », l'impact sur le stress et la performance au travail étant différent.

Comme toute recherche qualitative, ce travail présente plusieurs limites qui ouvrent autant de perspectives de recherche. Tout d'abord, les résultats de cette étude restent limités à l'entreprise considérée et aux personnes interrogées. Les conclusions que nous obtenons ne peuvent être généralisées. Il serait intéressant de renouveler ce type d'études dans des contextes professionnels différents afin de réduire la contingence éventuelle de ces résultats, tant en termes de variétés d'organisations que de secteurs d'activités. Cette recherche met par ailleurs en évidence des relations possibles entre différents concepts (exigences, ressources, stress, bien-être, performance, etc.) sans pouvoir étudier quantitativement l'existence, l'intensité et la 
causalité de ces relations. Une étude quantitative pourrait donc venir compléter et corroborer ces résultats encourageants.

\section{Références bibliographiques}

Abaidi-Ben Nasr, J. (2015), Le bien-être au travail : conception et validation d'une échelle de mesure, thèse de doctorat en sciences de gestion, université de La Rochelle.

Abord de Chatillon, E., \& Richard, D. (2015), «Du sens, du lien, de l'activité et de confort (SLAC) », Revue française de gestion, $\mathrm{n}^{\circ} 4$, p. 53-71.

Adobe (2015), Quarterly Digital Intelligence Briefing: Digital Trends 2015. URL : https://offers.adobe.com/en/uk/marketing/landings/econsultancy digital trends report 2 015.html.

Agence européenne pour la sécurité et la santé au travail (European Agency for Safety and Health at Work) (2002), How to Tackle Psychosocial Issues and Reduce Work-related Stress, factsheet 32, URL : https://osha.europa.eu/en/publications/factsheet-32-howtackle-psychosocial-issues-and-reduce-work-related-stress/view.

Al-Fudail, M., \& Mellar, H. (2008), « Investigating Teacher Stress When Using Technology », Computers \& Education, Vol. 51, n 3, p. 1103-1110.

Assadi, H., \& Denis, J. (2005), «Les usages de l'e-mail en entreprise : efficacité dans le travail ou surcharge informationnelle ?» in Le travail avec les technologies de l'information, E. Kessous et J.-L. Metzger (Eds), Hermes, Paris, p. 135-155.

Autissier, D., \& Lahlou, S. (1999), «Les limites organisationnelles des TIC: émergence d'un phénomène de saturation cognitive », in Actes de la 4 e conférence de l'AIM, p. 121-130.

Ayyagari, R. (2007), What and Why of Technostress: Technology Antecedents and Implication, thèse de doctorat, Clemson University.

Ayyagari, R., Grover, V., \& Purvis, R. (2011), « Technostress: technological antecedents and implications », MIS Quarterly, Vol. 35, $\mathrm{n}^{\circ}$ 4, p. 831-858.

Bakker, A. B., \& Demerouti, E. (2007), «The Job Demands-Resources Model: State of the Art », Journal of Managerial Psychology, Vol. 22, no 3, p. 309-328.

Bakker, A. B., Demerouti, E., De Boer, E., \& Schaufeli, W. B. (2003), « Job Demands and Job Resources as Predictors of Absence Duration and Frequency », Journal of Vocational Behavior, Vol. 62, nº 2, p. 341-356.

Beaudry, A., \& Pinsonneault, A. (2005), «Understanding user responses to information technology: A coping model of user adaptation », MIS Quarterly, Vol. 29, $\mathrm{n}^{\circ}$ 3, p. $493-$ 524.

Beehr, T. A., \& Newman, J. E., (1978), « Job stress, employee health, and organizational effectiveness: A facet analysis, model, and literature review », Personnel Psychology, Vol. 31, nº 4, p. 665-699.

Benedetto-Meyer, M., \& Klein, T. (2012), «Le rôle des TIC dans les reconfigurations des espaces et des temporalités : le brouillage des frontières entre travail et hors-travail » in L'impact des TIC sur les conditions de travail, T. Klein et D. Ratier (Eds), Centre d'analyse stratégique, p. 137-160.

Bérard, D. (2015), « L'irruption des TIC et l'éclatement de l'espace-temps du travail : travailler sans limites? » in Les cadres face aux TIC : Enjeux et risques psychosociaux au travail, C. Felio et L. Lerouge (Eds), L'Harmattan, p. 77-90.

Besseyre des Horts, C.-H., \& Isaac, H. (2006), « L'impact des TIC mobiles sur les activités des professionnels en entreprise ", Revue française de gestion, Vol. 9-10, $\mathrm{n}^{\text {os }} 168-169$, p. 243 263.

Biétry, F., \& Creusier, J. (2013), « Proposition d'une échelle de mesure positive du bien-être au travail (EPBET) », Revue de gestion des ressources humaines, Vol. 87, nº 1, p. 23-41. 
Boukef, N., \& Charki, M. H. (2019), « The Millefeuille theory revisited. New theoretical lenses to understand the Millefeuille effect », Systèmes d'information \& management, Vol. 24 $\mathrm{n}^{\mathrm{o}} 2$, p. $47-83$.

Bretesché, S., de Corbière, F., \& Geffroy B. (2012), « La messagerie électronique, principal métronome des activités de cadre »,La nouvelle revue du travail, URL: http://nrt.revues.org/262.

Centre d'analyse stratégique (2012), L'impact des TIC sur les conditions de travail, rapport coordonné par T. Klein et D. Ratier.

Cézard, M., \& Hamon-Cholet, S. (1999), Travail et charge mentale, synthèse $\mathrm{n}^{0} 27$, DARES, ministère de l'Emploi et de la Solidarité.

Chevallet, R., \& Moatty, F. (2012), «Impacts des TIC sur les rythmes, l'autonomie et le contrôle du travail » in L'impact des TIC sur les conditions de travail, T. Klein et D. Ratier (Eds), Centre d'analyse stratégique, p. 83-104.

Christopher, J. C. (1999), « Situating Psychological Well-Being: Exploring the Cultural Roots of Its Theory and Research », Journal of Counseling \& Development, Vol. 77, $\mathrm{n}^{\circ} 2$, p. 141152.

Cotton, P., \& Hart, P. M. (2003), « Occupational Wellbeing and Performance: A Review of Organisational Health Research », Australian Psychologist, Vol. 38, nº 2, p. 118-127.

Crawford, E. R., LePine, J. A., \& Rich, B. L. (2010), « Linking job demands and resources to employee engagement and burnout: a theoretical extension and meta-analytic test », Journal of Applied Psychology, Vol. 95, $\mathrm{n}^{0}$ 5, p. 834-848.

Cucchi, A. (2014), "Risques psycho-sociaux et TIC : le cas de l'usage des TIC à domicile », Actes du congrès de l'AIM 2014.

Dagenais-Desmarais, V. (2010), Du bien-être psychologique au travail : fondements théoriques, conceptualisation et instrumentation du construit, thèse de doctorat, université de Montréal.

Dagenais-Desmarais, V., \& Savoie, A. (2012), « What Is Psychological Well-Being, Really? A Grassroots Approach from the Organizational Sciences », Journal of Happiness Studies, Vol. 13, nº 4, p. 659-684.

Daniels, K. (2000), « Measures of Five Aspects of Affective Well-Being at Work », Human Relations, Vol. 53, nº 2, p. 275-294.

Davis, F. D., Bagozzi, R. P., \& Warshaw, P. R. (1989). «User acceptance of computer technology: a comparison of two theoretical models ", Management Science, Vol. 35, $\mathrm{n}^{\circ} 8$, p. 982-1003.

Day, A., Scott, N., \& Kelloway, E. K. (2010). « Information and communication technology: Implications for job stress and employee well-being » in New developments in theoretical and conceptual approaches to job stress, P. L. Perrewé et D. C. Ganster (Eds), Vol. 8, p. 317-350.

Demerouti, E., \& Bakker, A. B. (2011), « The Job Demands-Resources Model: Challenges for Future Research », SA Journal of Industrial Psychology, Vol. 37, n² 2, p. 1-9.

Demerouti, E., Bakker, A. B., Nachreiner, F., \& Schaufeli, W. B. (2001), « The Job DemandsResources Model of Burnout », Journal of Applied Psychology, Vol. 86, n 3, p. 499-512.

Demerouti, E., Le Blanc, P. M., Bakker, A. B., Schaufeli, W. B., \& Hox, J. (2009), « Present but Sick: A Three-Wave Study on Job Demands, Presenteeism and Burnout », Career Development International, Vol. 14, n ${ }^{\circ} 1$, p. 50-68.

Derks, D., Bakker, A. B., Peters, P., \& van Wingerden, P. (2016), « Work-related smartphone use, work-family conflict and family role performance: The role of segmentation preference », Human Relations, Vol. 69, nº 5, p. 1045-1068. 
Derks, D., Zecic, D., \& Bakker, A. B. (2010), Switching on and off: the impact of Smartphone use on employee well-being, 9th conference of the European academy of occupational health psychology, Rome.

Diener, E., \& Fujita, F. (1995), « Resources, Personal Strivings, and Subjective Well-Being: A Nomothetic and Idiographic Approach», Journal of Personality and Social Psychology, Vol. 68, nº 5, p. 926-935.

Ennis, L. A. (2005), "The Evolution of Technostress », Computers in Libraries, Vol. 25, $\mathrm{n}^{\circ} 8$, p. $10-12$.

Fallery, B., \& Rodhain, F. (2007), «Quatre approches pour l'analyse de données textuelles : lexicale, linguistique, cognitive, thématique ", in Actes de la $16^{e}$ conférence de l'Association internationale de management stratégique.

Farhoomand, A. F., \& Drury, D. H. (2002), « Managerial information overload», Communications of the ACM, Vol. 45, $\mathrm{n}^{\circ} 10, \mathrm{p} .127-131$.

Firestone, W. A. (1987), « Meaning in method: The rhetoric of quantitative and qualitative research », Educational Researcher, Vol. 16, n 7, p.16-21.

French, J. R. P., Caplan, R. D., \& Van Harrison, R. (1982), The mechanisms of job stress and strain, J. Wiley, New York.

Garo, J.-D. (2019), « Travail : « Le bureau est devenu le lieu de l'interruption permanente », Le Monde, 30 mars 2019.

Gheorghiu, M. D., \& Moatty, F. (2005), «Groupes sociaux et enjeux de la coopération au travail dans l'industrie », Réseaux, Vol. 23, nº 134, p. 91-122.

Godé-Sanchez, C. (2008), "Les TIC comme leviers du changement organisationnel : une analyse du cas des Armées américaines en Afghanistan », Systèmes d'information \& management, Vol. 13, $\mathrm{n}^{\mathrm{o}}$ 1, p. 7-30.

Gollac, M., Greenan, N., \& Hamon-Cholet, S. (2000), «L'informatisation de l'« ancienne » économie: nouvelles machines, nouvelles organisations et nouveaux travailleurs », Économie et statistique, Vol. 339-340, $\mathrm{n}^{\mathrm{o}}$ 1, p. 171-201.

Gomez, P.-Y., \& Chevallet, R. (2011), « Impacts des technologies de l'information sur la santé au travail », Revue française de gestion, Vol. 5, n² 214, p. 107-125.

Goodhue, D. L., \& Thompson, R. L. (1995), «Task-technology fit and individual performance », MIS Quarterly, Vol. 19, n 2, p. 213-236.

Greenan, N., Hamon-Cholet, S., Moatty, F., \& Rosanvallon, J. (2012), TIC et conditions de travail. Les enseignements de l'enquête COI, rapport du Centre d'étude de l'emploi.

GRePS-Apec (2011), L'impact des technologies de communication sur les cadres, rapport coordonné par M.-É. Bobillier Chaumon.

Grosjean, V. (2005), « Le bien-être au travail : un objectif pour la prévention », INRS - HST Cahiers de notes documentaires, $\mathrm{n}^{0} 198$, p. 29-40.

Gupta, A., Li, H., \& Sharda, R. (2013), «Should I Send this Message? Understanding the Impact of Interruptions, Social Hierarchy and Perceived Task Complexity on User Performance and Perceived Workload », Decision Support Systems, Vol. 55, n 1, p. 135145.

Habib, J., Béjean, M., \& Dumond, J. P. (2017), «Appréhender les transformations organisationnelles de la santé numérique à partir des perceptions des acteurs », Systèmes d'information \& management, Vol. 22, $\mathrm{n}^{\mathrm{0}} 1$, p. 39-69.

Hakanen, J. J., Schaufeli, W. B., \& Ahola, K. (2008), « The Job Demands-Resources Model: A Three-Year Cross-Lagged Study of Burnout, Depression, Commitment, and Work Engagement », Work \& Stress, Vol. 22, n 3, p. 224-241.

Harter, J. K., Schmidt, F. L., \& Hayes, T. L. (2002), « Business-Unit-Level Relationship Between Employee Satisfaction, Employee Engagement, and Business Outcomes: A MetaAnalysis », Journal of Applied Psychology, Vol. 87, nº 2, p. 268-279. 
Helmersen, P., Jalalian, A., Moran, G. \& Norman, F. (2001), Impacts of Information Overload, Eurescom.

Hudiburg, R. A., \& Necessary J. R. (1996), « Coping with Computer-Stress », Journal of Educational Computing Research, Vol. 15, nº 2, p. 113-124.

Hung, W. H., Chang, L. M., \& Lin, C. H. (2011), « Managing the Risk of Overusing Mobile Phones in the Working Environment: A Study of Ubiquitous Technostress », Proceedings of the Pacific Asia Conference on Information Systems.

Isaac, H., Campoy, É., \& Kalika, M. (2007), « Surcharge informationnelle, urgence et TIC. L'effet temporel des technologies de l'information », Management \& Avenir, Vol. 13, n 3, p. 149-168.

Joling, C., \& Kraan, K. (2008), Use of technology and working conditions in the European Union. European Foundation for the Improvement of Working and Living Conditions, Luxemburg.

Kalika, M., Charki, N. B., \& Isaac, H. (2007), « La théorie du millefeuille et l'usage des TIC dans l'entreprise ", Revue française de gestion, Vol. 3, p. 117-129.

Karasek, R. (1990), «Lower health risk with increased job control among white collar workers ", Journal of Organizational Behavior, Vol. 11, nº 3, p. 171-185.

Karasek Jr, R. A. (1979), « Job demands, job decision latitude, and mental strain: Implications for job redesign », Administrative Science Quarterly, Vol. 24, nº 2, p. 285-308.

Kaspar, J. (2012), Rapport de la Commission du Grand Dialogue de La Poste, Groupe la Poste.

Kefi, H., Mlaiki, A., \& Kalika, M. (2016), « Comprendre le phénomène de dépendance envers les réseaux sociaux numériques : les effets de l'habitude et de la surcharge informationnelle dans le cas de Facebook », Systèmes d'information \& management, Vol. 21, n ${ }^{\circ}$ 4, p.7-42.

Klein, T., \& Govaere, V. (2012), «Impacts des TIC sur le bien-être et la santé au travail» in L'impact des TIC sur les conditions de travail, T. Klein et D. Ratier (Eds), Centre d'analyse stratégique, p. 161-184.

Klein, T., \& Long, K. (2013), Conditions de travail, organisation du travail et usages des TIC selon les métiers. Une exploitation de l'enquête Conditions de travail, rapport $\mathrm{n}^{\circ} 2013-03$, Centre d'analyse stratégique, URL: http://archives.strategie.gouv.fr/cas/system/files/2013-03 conditions travail metiers.pdf.

Koolhaas, J. M., Bartolomucci, A., Buwalda, B., de Boer, S. F., Flügge, G., Korte, S. M., Meerlo, P., Murison, R., Olivier, B., Palanza, P., \& Richter-Levin, G. (2011), « Stress revisited: a critical evaluation of the stress concept », Neuroscience \& Biobehavioral Reviews, Vol. 35, no 5, p. 1291-1301.

Korunka, C., Zauchner, S., \& Weiss, A. (1997), « New Information Technologies, Job Profiles, and External Workload as Predictors of Subjectively Experienced Stress and Dissatisfaction at Work », International Journal of Human-Computer Interaction, Vol. 9, $\mathrm{n}^{\mathrm{o}} 4$, p. 407-424.

Lachmann, H., Larose C., \& Penicaud, M. (2010), Bien-être et efficacité au travail - 10 propositions pour améliorer la santé psychologique au travail, rapport fait à la demande du Premier ministre.

Lazarus, R. S., \& Folkman, S. (1984), Stress, appraisal, and coping, Springer Publishing Company Inc., New York.

Lee, K., \& Allen, N. J. (2002), «Organizational Citizenship Behavior and Workplace Deviance: The Role of Affect and Cognitions ", Journal of Applied Psychology, Vol. 87, $\mathrm{n}^{\mathrm{o}}$ 1, p. 131-142.

Lee, Y.-K., Chang, C.-T., Lin, Y., \& Cheng, Z.-H. (2014), « The dark side of smartphone usage: Psychological traits, compulsive behavior and technostress ", Computers in Human Behavior, Vol. 31, p. 373-383. 
Légeron, P. (2015), Le stress au travail, Odile Jacob, Paris.

Lowry, D., \& Moskos, M. (2005), Hanging on the mobile phone: Experiencing Work and Spatial Flexibility, Working Paper, National Institute of Labour Studies, Flinders University, Adelaide.

Lyubomirsky, S., Sheldon, K. M., \& Schkade, D. (2005, «Pursuing Happiness: The Architecture of Sustainable Change », Review of General Psychology, Vol. 9, $\mathrm{n}^{\circ}$ 2, p. 111131.

McGrath, J. E. (1976), « Stress and behavior in organizations », in Handbook of industrial and organizational psychology, M. D. Dunnette (Ed), Rand McNally College Pub. Co., Chicago.

Meijman, T. F., \& Mulder, G. (1998), « Psychological Aspects of Workload » in Handbook of Work and Organizational Psychology, P. J. D. Drenth, H. Thierry et C. de Wolff (Eds), Psychology Press, Hove.

Metzger, J.-L., \& Cléach, O. (2004), «Le télétravail des cadres : entre suractivité et apprentissage de nouvelles temporalités », Sociologie du travail, Vol. 46, nº 4, p. 433-450.

Miles, M. B., \& Huberman, A. M. (2003), Analyse des données qualitatives, De Boeck Supérieur.

Olivier de Sardan, J.-P. (1995), «La politique du terrain. Sur la production des données en anthropologie » in Enquête. Archives de la revue Enquête, ${ }^{\circ}$ 1, p. 71-109.

Oulasvirta, A., Rattenbury, T., Ma, L., \& Raita, E. (2012), « Habits make smartphone use more pervasive », Personal and Ubiquitous Computing, Vol. 16, $\mathrm{n}^{\circ}$ 1, p. 105-114.

Peyrat-Guillard, D., \& Samier, N. (2004), «TIC, implication et climat social : vers une autonomisation sous contraintes des salariés ", Revue de gestion des ressources humaines, Vol. 51, n ${ }^{0} 1$, p. 39-56.

Podsakoff, P. M., MacKenzie, S. B., Paine, J. B., \& Bachrach, D. G. (2000), « Organizational Citizenship Behaviors: A Critical Review of the Theoretical and Empirical Literature and Suggestions for Future Research », Journal of Management, Vol. 26, nº 3, p. 513-563.

Poole, C. E., \& Denny, E. (2001), «Technological Change in the Workplace: A Statewide Survey of Community College Library and Learning Resources Personnel », College \& Research Libraries, Vol. 62, nº 6, p. 503-515.

Ragu-Nathan, T. S., Tarafdar, M., Ragu-Nathan, B. S., \& Tu, Q. (2008), « The Consequences of Technostress for End Users in Organizations: Conceptual Development and Empirical Validation », Information Systems Research, Vol. 19, nº 4, p. 417-433.

Reix, R., \& Rowe, F. (2002), « La recherche en système d'information : de l'histoire au concept » in Faire de la recherche en systèmes d'information, F. Rowe (Ed), Paris, Vuibert, p. 3-20.

Rosen, L. D., \& Weil, M. M. (2000), Results of our 49-Month Study of Business Attitudes Show Clerical/Support Staff, Managers and Executives Using More Technology at Work and at Home and Becoming More Hesitant Toward New Technology, URL : http://www.csudh.edu/psych/49\%20Month\%20Study\%20of\%20Business\%20Attit udes\%20Toward\%20Technology.pdf.

Rupelle (de la), G., Fray, A.-M., \& Kalika, M. (2014), « Messagerie électronique, facteur de stress dans le cadre de la relation managériale », Revue de gestion des ressources humaines, Vol. 91, n ${ }^{\circ}$ 1, p. 13-28.

Ryan, R. M., \& Deci, E. L. (2001), « On happiness and human potentials: A review of research on hedonic and eudaimonic well-being », Annual Review of Psychology, Vol. 52, $\mathrm{n}^{0} 1$, p. 141-166.

Saintive, B. (2000), « Trois formes différenciées d'usage de la messagerie électronique au sein d'une organisation », Réseaux, Vol. 18, nº 104, p. 119-137. 
Schaufeli, W. B., \& Bakker, A. B. (2004), « Job demands, job resources, and their relationship with burnout and engagement: A multi-sample study », Journal of Organizational Behavior, Vol. 25, no 3, p. 293-315.

Schaufeli, W. B., \& Taris, T. W. (2014), «A critical review of the Job Demands-Resources Model: Implications for improving work and health» in Bridging occupational, organizational and public health, G. F. Bauer et O. Hämmig (Eds), Dordrecht, Springer, p. 43-68.

Schuler, R. S. (1980), "Definition and conceptualization of stress in organizations », Organizational Behavior and Human Performance, Vol. 25, $\mathrm{n}^{\circ}$ 2, p. 184-215.

Selye, H. (1973), « The Evolution of the Stress Concept: The originator of the concept traces its development from the discovery in 1936 of the alarm reaction to modern therapeutic applications of syntoxic and catatoxic hormones ", American Scientist, Vol. 61, $\mathrm{n}^{\mathrm{o}}$ 6, p. 692-699.

Shu, Q., Tu, Q., \& Wang, K. (2011), « The Impact of Computer Self-Efficacy and Technology Dependence on Computer-Related Technostress: A Social Cognitive Theory Perspective », International Journal of Human-Computer Interaction, Vol. 27, $\mathrm{n}^{\circ} 10$, p. 923-939.

Siegrist, J. (1996), « Adverse health effects of high-effort/low-reward conditions », Journal of Occupational Health Psychology, Vol. 1, n 1, p. 27-41.

Towers, I., Duxbury, L., Higgins, C., \& Thomas, J. (2006), « Time thieves and space invaders: Technology, work and the organization », Journal of Organizational Change Management, Vol. 19, no 5, p. 593-618.

Travaglianti, F. (2011), Smartphones : ressources ou contraintes pour l'employé? Analyse dans la perspective du JD-R model, mémoire de master, université de Liège.

Tu, Q., Wang, K., \& Shu, Q. (2005), «Computer-Related Technostress in China », Communications of the ACM, Vol. 48, $\mathrm{n}^{\mathrm{0}} 4$, p. 77-81.

Vaghefi, I., \& Lapointe, L., (2013), Can IT hurt productivity? An investigation of IT addiction, 34th International Conference on Information Systems, Milan.

Van den Broeck, A., De Cuyper, N., De Witte, H., \& Vansteenkiste, M. (2010), « Not all job demands are equal: Differentiating job hindrances and job challenges in the Job DemandsResources model », European Journal of Work and Organizational Psychology, Vol. 19, $\mathrm{n}^{\mathrm{o}} 6, \mathrm{p} .735-759$.

Venin, T. (2013), Techniques de l'information et de la communication et risques psychosociaux sur le poste de travail tertiaire, thèse de doctorat, université de Pau et des Pays de l'Adour.

Wang, K., Shu, Q., \& Tu, Q. (2008), «Technostress Under Different Organizational Environments: An Empirical Investigation », Computers in Human Behavior, Vol. 24, $\mathrm{n}^{\mathrm{o}} 6$, p. 3002-3013.

Wanlin, P. (2007), « L'analyse de contenu comme méthode d'analyse qualitative d'entretiens: une comparaison entre les traitements manuels et l'utilisation de logiciels », Recherches qualitatives, hors-série, $\mathrm{n}^{\mathrm{0}} 3$, p. 243-272.

Warr, P. (1990), "The Measurement of Well-Being and Other Aspects of Mental Health », Journal of Occupational Psychology, Vol. 63, nº 3, p. 193-210.

Webtorials (2017), Workplace Productivity and Communications Technology Report, URL: http://www.webtorials.com/main/resource/papers/Mitel/paper2/2017-WorkplaceProductivity-SotM.pdf.

Wright, T. A., Cropanzano, R., Denney, P. J., \& Moline, G. L. (2002), « When a Happy Worker Is a Productive Worker: A Preliminary Examination of Three Models », Canadian Journal of Behavioural Science / Revue canadienne des sciences du comportement, Vol. 34, $\mathrm{n}^{\circ} 3$, 146. 
Yin, R. K. (2017), Case Study Research and Applications: Design and Methods, Sage Publications. 


\begin{tabular}{|c|c|c|}
\hline $\begin{array}{c}\text { Trame } \\
\text { d'entretien }\end{array}$ & Questions & $\begin{array}{l}\text { Objectifs de recherche / thèmes } \\
\text { d'analyse }\end{array}$ \\
\hline \multirow{3}{*}{ Introduction } & \multirow{3}{*}{$\begin{array}{l}\text { Étude sur l'usage des technologies } \\
\text { qui ont été déployées et sur la vie } \\
\text { au travail }\end{array}$} & $\begin{array}{l}\text { Se présenter, remercier la personne pour } \\
\text { sa disponibilité et préciser les règles de } \\
\text { déontologie }\end{array}$ \\
\hline & & Explication du contexte de l'étude \\
\hline & & $\begin{array}{l}\text { Rappel de l'objectif de l'entretien et du } \\
\text { rôle de l'interviewé }\end{array}$ \\
\hline $\begin{array}{l}\text { Caractéristiques } \\
\text { personnelles }\end{array}$ & $\begin{array}{l}\text { Pourriez-vous me décrire votre } \\
\text { métier? }\end{array}$ & \\
\hline \multirow{2}{*}{$\begin{array}{l}\text { Présentation de } \\
\text { la technologie }\end{array}$} & $\begin{array}{l}\text { De quelle technologie avez-vous } \\
\text { été équipé (téléphone intelligent, } \\
\text { tablette, ordinateur portable)? }\end{array}$ & $\begin{array}{l}\text { Pour quelles fonctionnalités prévues } \\
\text { (prise de rendez-vous, accessibilité aux } \\
\text { mails, démarches commerciales, ...) }\end{array}$ \\
\hline & $\begin{array}{l}\text { Ce genre d'outil vous était-il déjà } \\
\text { familier? (Privé ou prof.) }\end{array}$ & Pour juger du niveau de réticence \\
\hline \multirow{2}{*}{$\begin{array}{l}\text { Perception du } \\
\text { changement }\end{array}$} & $\begin{array}{l}\text { Comment avez-vous réagi au } \\
\text { déploiement de la TN? }\end{array}$ & Quelle utilisation initiale prévue \\
\hline & $\begin{array}{l}\text { Selon vous, quelles étaient les } \\
\text { motivations de la direction? }\end{array}$ & $\begin{array}{l}\text { Motivation vis-à-vis de l'individu, de } \\
\text { l'organisation }\end{array}$ \\
\hline \multirow{7}{*}{ Usages des TN } & \multirow{2}{*}{$\begin{array}{l}\text { Comment s'est passée la prise en } \\
\text { main de la technologie? }\end{array}$} & Consultation préalable des utilisateurs \\
\hline & & Formation \\
\hline & \multirow{5}{*}{$\begin{array}{l}\text { Quelle est votre utilisation } \\
\text { actuelle de la TN ? (Depuis } \\
\text { combien de temps l'utilisez- } \\
\text { vous?) }\end{array}$} & Appropriation \\
\hline & & Limites et apports de la $\mathrm{TN}$ \\
\hline & & Détournements d'usages \\
\hline & & Usages non conformes (privé) \\
\hline & & Adaptation à l'organisation \\
\hline \multirow{6}{*}{$\begin{array}{l}\text { BET et } \\
\text { technostress }\end{array}$} & \multirow{6}{*}{$\begin{array}{l}\text { Votre métier a-t-il changé depuis } \\
\text { le déploiement de cette } \\
\text { technologie? }\end{array}$} & Relations avec les collègues \\
\hline & & $\begin{array}{l}\text { Relations avec la hiérarchie (contrôle, } \\
\text { reconnaissance) }\end{array}$ \\
\hline & & $\begin{array}{l}\text { Changement de communication (accès } \\
\text { mail) }\end{array}$ \\
\hline & & Modification espaces de travail \\
\hline & & Conflit travail / famille \\
\hline & & Changement des horaires de travail \\
\hline
\end{tabular}


Travail en équipe

Apprentissage, formation

Reconnaissance et appréciation du travail

Pour vous, que représente le bienêtre au travail ?
BET et technostress

Avez-vous le sentiment que votre

vie au travail a évolué ces derniers temps et, si oui, qu'est-ce qui a influencé cela selon vous?

Pensez-vous que l'arrivée de ce $\mathrm{TN}=$ stress, BET ou les deux nouvel outil a modifié votre BET ?

Responsabilités

Mobilisation de compétences individuelles et par équipe

Objectifs et finalité de travail clairement identifiés

Environnement de travail et pratique sociale de l'entreprise

Pensez-vous que l'arrivée de la $\mathrm{TN}$ ait déplacé la frontière entre vie professionnelle et vie privée?

Votre entourage vous a-t-il fait remarquer que vous aviez changé depuis le déploiement de la TN ?

Pensez-vous l'arrivée de la TN dans votre travail a modifié votre bien-être général ?

Souhaitez-vous aborder un point en particulier que nous n'aurions pas abordé et qui vous semble

Conclusion important?

Y a-t-il des gens que je pourrais rencontrer qui auraient des choses à raconter sur ce sujet ?
Conflit travail-famille

Sentiment de fatigue en rentrant du travail

Vie professionnelle empêchant de passer du temps avec la famille

Fatigue ressentie au travail à cause des activités personnelles

Préoccupations personnelles empiétant sur la vie professionnelle

\section{Remerciements}

Détail sur la suite de l'étude (validation de la retranscription, explication phase d'analyse)

Intérêt pour les interviewés et l'entreprise 
Annexe 2 - Arbre de codage de l'étude

\begin{tabular}{|c|c|c|}
\hline $\begin{array}{l}\text { Nœuds } \\
\text { primaires }\end{array}$ & Nœuds secondaires & $\begin{array}{l}\text { Description ou exemples de } \\
\text { regroupement pour l'analyse }\end{array}$ \\
\hline Cas & Noms des personnes interrogées & $\begin{array}{l}\text { Importation de l'ensemble des cas } \\
\text { (entretiens réalisés) }\end{array}$ \\
\hline Métier & & $\begin{array}{l}\text { Description générale du métier des } \\
\text { personnes interrogées }\end{array}$ \\
\hline \multirow{3}{*}{ Usage des TN } & Règles d'usage & \multirow{3}{*}{$\begin{array}{l}\text { Ensemble des questions relatives aux } \\
\text { usages. Les nœuds « règles d'usage » et } \\
\text { « utilisation des TN » ont été finalement } \\
\text { regroupés lors de l'analyse }\end{array}$} \\
\hline & Utilisation des TN & \\
\hline & Usage excessif (addiction) & \\
\hline \multirow{8}{*}{$\begin{array}{l}\text { Impact des TN } \\
\text { sur le métier }\end{array}$} & Chronophage - temps morts & $\begin{array}{l}\text { Création de temps morts et pertes de } \\
\text { temps liées aux TN }\end{array}$ \\
\hline & Conciliation travail-famille & \multirow{2}{*}{$\begin{array}{l}\text { Regroupement de ces deux nœuds avec } \\
\text { «Modification des espaces et temps de } \\
\text { travail» }\end{array}$} \\
\hline & $\begin{array}{l}\text { Conciliation - remarques de } \\
\text { l'entourage }\end{array}$ & \\
\hline & Efficacité au travail & $\begin{array}{l}\text { Influence des TN sur l'efficacité au } \\
\text { travail }\end{array}$ \\
\hline & $\begin{array}{l}\text { Modification des espaces et temps } \\
\text { de travail }\end{array}$ & $\begin{array}{l}\text { Regroupement de ce nœud avec les } \\
\text { nœuds «Conciliation travail-famille » et } \\
\text { «conciliation - remarques de } \\
\text { l'entourage» }\end{array}$ \\
\hline & Pression liée aux TN & \multirow{2}{*}{$\begin{array}{l}\text { Regroupement de ces deux nœuds pour } \\
\text { analyser la pression engendrée par les } \\
\text { TN sur le métier }\end{array}$} \\
\hline & $\begin{array}{l}\text { Réactivité et pression de la } \\
\text { réponse }\end{array}$ & \\
\hline & Satisfaction au travail & $\begin{array}{l}\text { Les TN comme vecteurs de satisfaction } \\
\text { au travail }\end{array}$ \\
\hline \multirow{5}{*}{$\begin{array}{l}\text { Définition du } \\
\text { bien-être au } \\
\text { travail }\end{array}$} & Ambiance de travail & \multirow{5}{*}{$\begin{array}{l}\text { Ces nœuds ont permis d'identifier les } \\
\text { manifestations du bien-être au travail } \\
\text { pour les répondants, en lien avec les TN. } \\
\text { Ce sont des nœuds transversaux qui ont } \\
\text { servi à préciser les analyses issues des } \\
\text { autres nœuds }\end{array}$} \\
\hline & $\begin{array}{l}\text { Équilibre vie privée-vie } \\
\text { professionnelle }\end{array}$ & \\
\hline & Formation et apprentissage & \\
\hline & Mobilisation de compétences & \\
\hline & Reconnaissance & \\
\hline
\end{tabular}


Sens au travail

Relations avec les collègues et la hiérarchie

$\mathrm{TN}$, stress et bien-être au travail
Influence des TN sur le bien-être au travail

Influence des TN sur le stress
Nœuds de synthèse qui reprennent en partie les nœuds précédents en insistant sur les dimensions stress et bien-être 